\title{
Research Article \\ Exponential Estimates and Stabilization of Discrete-Time Singular Time-Delay Systems Subject to Actuator Saturation
}

\author{
Jinxing $\operatorname{Lin}^{1,2}$ \\ ${ }^{1}$ College of Automation, Nanjing University of Posts and Telecommunications, Nanjing 210003, China \\ ${ }^{2}$ Key Laboratory of Measurement and Control of Complex Systems of Engineering, Ministry of Education, \\ Southeast University, Nanjing 210096, China \\ Correspondence should be addressed to Jinxing Lin, jxlin2004@126.com
}

Received 29 February 2012; Revised 20 June 2012; Accepted 24 July 2012

Academic Editor: Recai Kilic

Copyright (c) 2012 Jinxing Lin. This is an open access article distributed under the Creative Commons Attribution License, which permits unrestricted use, distribution, and reproduction in any medium, provided the original work is properly cited.

\begin{abstract}
This paper is concerned with exponential estimates and stabilization of a class of discrete-time singular systems with time-varying state delays and saturating actuators. By constructing a decay-rate-dependent Lyapunov-Krasovskii function and utilizing the slow-fast decomposition technique, an exponential admissibility condition, which not only guarantees the regularity, causality, and exponential stability of the unforced system but also gives the corresponding estimates of decay rate and decay coefficient, is derived in terms of linear matrix inequalities (LMIs). Under the proposed condition, the exponential stabilization problem of discrete-time singular time-delay systems subject actuator saturation is solved by designing a stabilizing state feedback controller and determining an associated set of safe initial conditions, for which the local exponential stability of the saturated closed-loop system is guaranteed. Two numerical examples are provided to illustrate the effectiveness of the proposed results.
\end{abstract}

\section{Introduction}

Singular time-delay systems (STDSs) arise naturally in many engineering fields such as electric networks, chemical processes, lossless transmission lines, and so forth [1]. A STDS is a mixture of delay differential equations and delay difference equations; such a complex nature of STDS leads to abundant dynamics, for example, non-strictly proper transcendental equations, irregularity, impulses or non-causality. Therefore, the study of such systems is much more complicated than that for normal state-space time-delay systems. In the past two decades, a great number of stability results on STDSs have been reported in the literature; see, for example, [2-8] and the references therein. 
It is noted that many stability results for STDSs are concerned with asymptotic stability. Practically, however, exponential stability is more important because the transient process of a system can be described more clearly once the decay rate is determined [9]. Therefore, in recent years, the study of exponential estimates problem of STDSs has received increasing attention, and a few approaches have been proposed. For example, in [10,11], the STDS was decomposed into slow (differential) and fast (algebraic) subsystems and the exponential stability of the slow subsystem was proved by using the Lyapunov method. Subsequently, the solutions of the fast subsystem was bounded by an exponential term using a function inequality. However, this approach cannot give an estimate of the convergence rate of the system. To overcome this difficulty, Shu and Lam [12] and Lin et al. [13] adopted the Lyapunov-Krasovskii function method $[14,15]$ and some improvements have been obtained. In $[16,17]$, an exponential estimates approach for SSTDs was presented by employing the graph theory to establish an explicit expression of the state variables of fast subsystem in terms of those of slow subsystem and the initial conditions, which allows to prove the exponential stability of the fast subsystem. However, all of the above results are related to continuous-time STDSs. To the best of the authors' knowledge, the problem of exponential estimates of discrete-time STDSs has not been investigated yet. One possible reason is the difficulty in obtaining the estimates for solutions of the corresponding fast subsystem. Therefore, the first aim is to develop effective approach to give the exponential estimates of discrete-time STDSs.

On the other hand, actuator saturation is also an important phenomenon arising in engineering. Saturation nonlinearity not only deteriorates the performance of the closedloop systems but also is the source of instability. Stabilization of normal state-space systems subject to actuator saturation has therefore attracted much attention from many researchers; see, for example, [18-22], and the references cited therein. Recently, some results for normal state-space systems have been generalized to singular systems. For example, semiglobal stabilization and output regulation of continuous-time singular system subject to input saturation were addressed in [23] by assuming that the open-loop system is semistable and impulse free which allows a state transformation such that the singular system is transformed into a normal system. Also, an algebraic Riccati equation approach to semiglobal stabilization of discrete-time singular linear systems with input saturation was proposed in [24] without any transformation of the original singular system. The invariant set approach developed for state-space system in [18] was extended to general, not necessarily semistable, continuoustime singular system in [25]. This approach was further extended to the analysis of the $\mathcal{L}_{2}$ gain and $\mathcal{L}_{\infty}$ performance for continuous-time singular systems under actuator saturation [26] and the analysis and design of discrete-time singular systems under actuator saturation $[27,28]$, respectively. In [17], estimation of domain of attraction for continuous-time STDSs with actuator saturation and the design of static output feedback controller that maximize it were proposed. However, so far, few work exists to address the stabilization problem for discrete-time STDSs subject to actuator saturation, which forms the second object of this paper.

In this paper, we investigate the problems of exponential estimates and stabilization for a class of discrete-time singular systems with time-varying delays and saturating actuators. The main contributions of the paper are twofold:

(1) In terms of linear matrix inequalities (LMIs), an exponential admissibility condition, which not only guarantees the regularity, causality and exponential stability of the unforced system but also gives the corresponding estimates of 
decay rate and decay coefficient, is derived by constructing a decay-rate-dependent Lyapunov-Krasovskii function and using the slow-fast decomposition.

(2) The exponential stabilization problem of STDSs with saturating actuators is solved by designing a stabilizing state feedback controller and determining an associated set of safe initial conditions, for which the local exponential stability of the saturated closed-loop system is guaranteed. The existence criterion of the desired controller is formulated, and an LMI optimization approach is proposed to enlarge the domain of safe initial conditions.

The paper is organized as follows. Problem statement and the preliminaries are given in Section 2. In Section 4, we present the exponential estimates for the STDSs and the solutions to the stabilization problem for the system with saturating actuators. Numerical examples will be given in Section 4 to illustrate the effectiveness of the proposed method. The paper will be concluded in Section 5 .

Notation. For real symmetric matrices $P, P>0(P \geq 0)$ means that matrix $P$ is positive definite (semipositive definite). $\lambda_{\max }(P)\left(\lambda_{\min }(P)\right)$ denotes the largest (smallest) eigenvalue of the positive definite matrix $P$. $\mathbf{R}^{n}$ is the $n$-dimensional real Euclidean space and $\mathbf{R}^{m \times n}$ is the set of all real $m \times n$ matrices. $\mathbf{Z}^{+}$represents the sets of all non-negative integers. The superscript " $T$ " represents matrix transposition, and " $*$ " in a matrix is used to represent the term which is induced by symmetry. $\operatorname{diag}\{\cdots\}$ stands for a block-diagonal matrix. $\operatorname{Sym}\{A\}$ is the shorthand notation for $A+A^{T}$. For two integers $n_{1}$ and $n_{2}$ with $n_{1} \leq n_{2}$, we use $\mathbf{I}\left[n_{1}, n_{2}\right]$ to denote the integer set $\left\{n_{1}, n_{1}+1, \ldots, n_{2}\right\}$. Let $\mathcal{C}_{n, d}=\left\{\phi: \mathbf{I}[-d, 0] \rightarrow \mathbf{R}^{n}\right\}$ denote the Banach space of family continuous vector valued functions mapping the interval $\mathbf{I}[-d, 0]$ to $\mathbf{R}^{n}$ with the topology of uniform convergence. Denote $x_{k}(s)=x(k+s), \forall s \in \mathbf{I}[-d, 0] .\|\cdot\|$ refers to either the Euclidean vector norm or the induced matrix two-norm. For a function $\phi \in \mathcal{C}_{n, d}$, its norm is defined as $\|\phi\|_{c}=\sup _{s \in \mathrm{I}[-d, 0]}\|\phi(s)\|$.

\section{Problem Statement and Preliminaries}

\subsection{Problem Statement}

Consider a class of discrete-time singular system subject to time-varying delay and actuator saturation as follows:

$$
\begin{gathered}
E x(k+1)=A x(k)+A_{d} x(k-d(k))+B \operatorname{sat}(u(k)), \\
x(s)=\phi(s), \quad s \in \mathbf{I}[-\bar{d}, 0],
\end{gathered}
$$

where $x(k) \in \mathbf{R}^{n}$ is the system state, $u(k) \in \mathbf{R}^{m}$ is the control input, and $\phi(s) \in \mathcal{C}_{n, \bar{d}}$ is a compatible vector valued initial function. $d(k)$ is a time-varying delay satisfying $0<$ $\underline{d} \leq d(k) \leq \bar{d}$, where $\underline{d}$ and $\bar{d}$ are constant positive scalars representing the minimum and maximum delays, respectively. The matrix $E$ is singular and rank $E=r<n . A, A_{d}$ and $B$ are known constant matrices. The function sat $: \mathbf{R}^{m} \rightarrow \mathbf{R}^{m}$ is the standard saturation function defined as $\operatorname{sat}(u(k))=\left[\operatorname{sat}\left(u_{1}(k)\right) \operatorname{sat}\left(u_{2}(k)\right) \cdots \operatorname{sat}\left(u_{m}(k)\right)\right]^{T}$, where $\operatorname{sat}\left(u_{i}(k)\right)=$ $\operatorname{Sign}\left(u_{i}(k)\right) \min \left\{1,\left|u_{i}(k)\right|\right\}$. Note that the notation of sat $(\cdot)$ is slightly abused to denote scalar values and vector valued saturation functions. Also note that it is without loss of generality to assume unity saturation level [20]. 
In this paper, we consider the design of a linear state feedback control law of the following form [27]:

$$
u(k)=F x(k), \quad F=\bar{F} E,
$$

where $F \in \mathbf{R}^{m \times n}$ and $\bar{F} \in \mathbf{R}^{m \times n}$. The closed-loop system under this feedback is given by

$$
E x(k+1)=A x(k)+A_{d} x(k-d(k))+B \operatorname{sat}(\bar{F} E x(k)) .
$$

Remark 2.1. The state feedback of the form (2.2) is used to guarantee the uniqueness of the solution of system (2.1). For that, since rank $E=r<n$, there exist two nonsingular matrices $G, H \in \mathbf{R}^{n \times n}$ such that

$$
\begin{gathered}
G E H=\left[\begin{array}{cc}
I_{r} & 0 \\
0 & 0
\end{array}\right], \quad G A H=\left[\begin{array}{ll}
A_{11} & A_{12} \\
A_{21} & A_{22}
\end{array}\right], \quad G A_{d} H=\left[\begin{array}{ll}
A_{d 11} & A_{d 12} \\
A_{d 21} & A_{d 22}
\end{array}\right], \\
G B=\left[\begin{array}{l}
B_{1} \\
B_{2}
\end{array}\right], \quad F H=\left[\begin{array}{ll}
F_{1} & F_{2}
\end{array}\right], \quad \bar{F} G^{-1}=\left[\begin{array}{ll}
\bar{F}_{1} & \bar{F}_{2}
\end{array}\right], \\
x(k)=H\left[\begin{array}{l}
x_{1}(k) \\
x_{2}(k)
\end{array}\right], \quad x_{1}(k) \in \mathbf{R}^{r}, \quad x_{2}(k) \in \mathbf{R}^{n-r} .
\end{gathered}
$$

If the state feedback is taken as the general form, that is, $u(k)=F x(k)$, by using (2.4), then system (2.3) is restricted system equivalent (r.s.e.) to the following one:

$$
\begin{gathered}
x_{1}(k+1)=A_{11} x_{1}(k)+A_{12} x_{2}(k)+A_{d 11} x_{1}(k-d(k))+A_{d 12} x_{2}(k-d(k)) \\
+B_{1} \operatorname{sat}\left(F_{1} x_{1}(k)+F_{2} x_{2}(k)\right), \\
0=A_{21} x_{1}(k)+A_{22} x_{2}(k)+A_{d 21} x_{1}(k-d(k))+A_{d 22} x_{2}(k-d(k))+B_{2} \operatorname{sat}\left(F_{1} x_{1}(k)+F_{2} x_{2}(k)\right) .
\end{gathered}
$$

From the second equation of (2.5), it can be seen that $x_{2}(k)$ is in the function sat $(\cdot)$. Hence, if $B_{2} \neq 0$, for given $x_{1}(0)$, the solution of $x_{2}(0)$ is not unique even $A_{22}$ is nonsingular. However, if $u(k)=\bar{F} E x(k)$, then system (2.3) is r.s.e. to

$$
\begin{gathered}
x_{1}(k+1)=A_{11} x_{1}(k)+A_{12} x_{2}(k)+A_{d 11} x_{1}(k-d(k))+A_{d 12} x_{2}(k-d(k))+B_{1} \operatorname{sat}\left(\bar{F}_{1} x_{1}(k)\right), \\
0=A_{21} x_{1}(k)+A_{22} x_{2}(k)+A_{d 21} x_{1}(k-d(k))+A_{d 22} x_{2}(k-d(k))+B_{2} \operatorname{sat}\left(\bar{F}_{1} x_{1}(k)\right),
\end{gathered}
$$

which implies that, for given $x_{1}(k)$, the unique solution of $x_{2}(k)$ can be obtained when $A_{22}$ is nonsingular. Nevertheless, it should be pointed that the state feedback (2.2) may be conservative due to its special structure. 

definitions.

To describe the main objective of this paper more precisely, we introduce the following

Definition 2.2 (see [29]). System $E x(k+1)=A x(k)$ (or the pair $(E, A)$ ) is said to be regular if $\operatorname{det}(z E-A)$ is not identically zero, and if $\operatorname{deg}(\operatorname{det}(z E-A))=\operatorname{rank} E$, then it is further said to be causal.

Definition 2.3 (see [6]). System (2.1) with $u(k)=0$ is said to be regular and causal, if the pair $(E, A)$ is regular and causal.

Note that regularity and causality of system (2.1) with $u(k)=0$ ensure that the solution to this system exists and is unique for any given compatible initial value $\phi(s)$.

Definition 2.4. System (2.1) under feedback law (2.2) is said to be exponentially stable with decay rate $\lambda(\lambda>1)$ if, for any compatible initial conditions $x_{k_{0}}(s)=x\left(k_{0}+s\right), s \in \mathbf{I}[-\bar{d}, 0]$, its solution $x\left(k, x_{k_{0}}\right)$ satisfies $\left\|x\left(k, x_{k_{0}}\right)\right\| \leq \epsilon \lambda^{-\left(k-k_{0}\right)}\left\|x_{k_{0}}\right\|_{c}$ for all $k \geq k_{0}$, where $\left\|x_{k_{0}}\right\|_{c}=$ $\sup _{s \in \mathrm{I}[-\bar{d}, 0]}\left\|x\left(k_{0}+s\right)\right\|, k_{0}$ is the initial time step, and $\epsilon>0$ is the decay coefficient.

We are interested in the exponential estimates and design for system (2.3). For any compatible initial condition $x_{0}=\phi \in \mathcal{C}_{n, \bar{d}}$, denote the state trajectory of system (2.1) as $x\left(k, x_{0}\right)$; then the domain of attraction of the origin is

$$
\mathcal{S}=\left\{\phi \in \mathcal{C}_{n, \bar{d}}: \lim _{k \rightarrow \infty} x\left(k, x_{0}\right)=0\right\}
$$

In general, for a given stabilizing state feedback gain $\bar{F}$, it is impossible to determine exactly the domain of attraction of the origin with respect to system (2.3). Therefore, the purpose of this paper is to design a state feedback gain $\bar{F}$ and determine a suitable set of initial condition $\Phi=\left\{\phi \in C_{n, \bar{d}}:\|\phi\|_{c}^{2} \leq \delta\right\} \in \mathcal{S}$ from which the regularity, causality, and exponential stability of the closed-loop system (2.3) is ensured. Also, we are interested in maximizing the size of this set, that is, obtaining the maximal value of $\delta$.

\subsection{Preliminary Results}

Define

$$
\begin{aligned}
& \zeta(k)=\left[\begin{array}{llll}
x^{T}(k) & x^{T}(k-d(k)) & x^{T}(k-\underline{d}) & x^{T}(k-\bar{d})
\end{array}\right]^{2}, \\
& y(k)=x(k+1)-x(k) .
\end{aligned}
$$


Lemma 2.5. For any appropriately dimensioned matrices $R>0$ and $N$, two positive time-varying integer $d\left(k_{1}\right)$ and $d\left(k_{2}\right)$ satisfying $d\left(k_{1}\right)+1 \leq d\left(k_{2}\right) \leq \bar{d}$, and a scalar $\lambda>0$, the following equality holds

$$
\begin{aligned}
& -\sum_{l=k-d\left(k_{2}\right)}^{k-d\left(k_{1}\right)-1} y^{T}(l) E^{T} \lambda^{k-l} R E y(l) \\
& =c \zeta^{T}(k) N R^{-1} N^{T} \zeta(k)+2 \zeta^{T}(k) N \sum_{l=k-d\left(k_{2}\right)}^{k-d\left(k_{1}\right)-1} E y(l) \\
& \quad-\sum_{l=k-d\left(k_{2}\right)}^{k-d\left(k_{1}\right)-1}\left(\zeta^{T}(k) N+\lambda^{k-l} y^{T}(l) E^{T} R\right)\left(\lambda^{k-l} R\right)^{-1}\left(N^{T} \zeta_{k}+\lambda^{k-l} R E y(l)\right),
\end{aligned}
$$

where $c=\left(\lambda^{-d\left(k_{2}\right)}-\lambda^{-d\left(k_{1}\right)}\right) /(1-\lambda)$.

Proof. See the Appendix.

Lemma 2.6 (see [30]). Given a matrix $D$, let a positive-definite matrix $S$ and a positive scalar $\eta \in$ $(0,1)$ exist such that

$$
D^{T} S D-\eta^{2} S<0
$$

then the matrix $D$ satisfies the bound

$$
\left\|D^{i}\right\| \leq x e^{-\lambda i}, \quad i=0,1, \ldots,
$$

where $x=\sqrt{\lambda_{\max }(S) / \lambda_{\min }(S)}$ and $\lambda=-\ln (\eta)$.

Lemma 2.7. Let $0<\underline{d} \leq d(k) \leq \bar{d}$. Consider the following system:

$$
x(k)+D x(k-d(k))=f(k), \quad k \geq 0,
$$

where $\left\|D^{i}\right\| \leq x e^{-\lambda i}, \lambda>0, i=0,1, \ldots$, and $\|f(k)\| \leq \kappa e^{-\beta k}, k \geq 0$. If

$$
\beta \bar{d}-\lambda<0
$$

Then, for any compatible initial function $\phi \in \mathcal{C}_{n, \bar{d}}$, the solution $x(k, \phi)$ of $(2.10)$ satisfies

$$
\|x(k, \phi)\| \leq\left(x\|\phi\|_{c}+x \kappa \frac{1}{1-e^{\beta \bar{d}-\lambda}}+\kappa\right) e^{-r k}, \quad k \geq 0
$$

where $r=\min \{\lambda / \bar{d}, \beta\}$.

Proof. See the Appendix. 
Discrete Dynamics in Nature and Society

For a matrix $F \in \mathbf{R}^{m \times n}$, denote the $j$ th row of $F$ as $f_{j}$ and define

$$
\mathcal{L}(F)=\left\{x(k) \in \mathbf{R}^{n}:\left|f_{j} x(k)\right| \leq 1, j \in \mathbf{I}[1, m]\right\} .
$$

Let $P \in \mathbf{R}^{n \times n}$ be a positive-definite matrix and $E^{T} P E \geq 0$, the set $\Omega\left(E^{T} P E, 1\right)$ is defined by

$$
\Omega\left(E^{T} P E, 1\right)=\left\{x(k) \in \mathbf{R}^{n}: x^{T}(k) E^{T} P E x(k) \leq 1\right\} .
$$

Also, let $\mho$ be the set of $m \times m$ diagonal matrices whose diagonal elements are either 1 or 0 . There are $2^{m}$ elements in $\mho$. Suppose that each element of $\mho$ is labeled as $D_{i}, i \in \mathbf{I}\left[1,2^{m}\right]$ and denote $D_{i}^{-}=I-D_{i}$. Clearly, $D_{i}$ is also an element of $\mho$ if $D_{i} \in \mho$.

Lemma 2.8 (see [18]). Let $F, H \in \mathbf{R}^{m \times n}$ be given. If $x(k) \in \mathcal{L}(H)$, then $\operatorname{sat}(F x(k)$ can be expressed as

$$
\operatorname{sat}(F x(k))=\sum_{i=1}^{2^{m}} \alpha_{i}(k)\left(D_{i} F+D_{i}^{-} H\right) x(k),
$$

where $\alpha_{i}(k)$ for $i \in \mathbf{I}\left[1,2^{m}\right]$ are some variables satisfying $\alpha_{i}(k) \geq 0$ and $\sum_{i=1}^{2^{m}} \alpha_{i}(k)=1$.

Lemma 2.9 (see [31]). Given matrices $X, Y$, and $Z$ with appropriate dimensions, and $Y$ is symmetric. Then there exists a scalar $\rho>0$, such that $\rho I+Y>0$ and $-\operatorname{Sym}\left\{X^{T} Z\right\}-Z^{T} Y Z \leq$ $X^{T}(\rho I+Y)^{-1} X+\rho Z^{T} Z$.

\section{Main Results}

In this section, we will first present a delay-dependent LMI condition which guarantees the regularity, causality and exponential stability of the unforced system (2.1) (i.e., with $u(k)=0$ ) with a predefined decay rate.

Theorem 3.1. Given constants $0<\alpha<1$ and $0<\underline{d}<\bar{d}$. If there exist symmetric matrices $X>0$, $Q_{l}>0, l=1,2,3, Z_{v}>0, v=1,2$ and $S$, and matrices $M=\left[\begin{array}{llll}M_{1}^{T} & M_{2}^{T} & M_{3}^{T} & M_{4}^{T}\end{array}\right]^{T}, N=$ $\left[\begin{array}{llll}N_{1}^{T} & N_{2}^{T} & N_{3}^{T} & N_{4}^{T}\end{array}\right]^{T}$ and $T=\left[\begin{array}{llll}T_{1}^{T} & T_{2}^{T} & T_{3}^{T} & T_{4}^{T}\end{array}\right]^{T}$ such that the following inequality holds

$$
\left[\begin{array}{ll}
\Phi & \Psi \\
* & \Gamma
\end{array}\right]<0
$$


where

$$
\begin{gathered}
\Phi=\operatorname{diag}\left\{-(1-\alpha) E^{T} X E+(1+\tilde{d}) Q_{1}+Q_{2}+Q_{3},-(1-\alpha)^{\bar{d}} Q_{1},-(1-\alpha)^{\underline{d}} Q_{2},-(1-\alpha)^{\bar{d}} Q_{3}\right\} \\
+\operatorname{Sym}\left\{M \Lambda_{1}+N \Lambda_{2}+T \Lambda_{3}\right\}+\mathcal{A}_{1}^{T} X \mathcal{A}_{1}-\mathcal{A}_{1}^{T} R^{T} S R \mathcal{A}_{1}+\mathcal{A}_{2}^{T} U \mathcal{A}_{2}, \\
\Psi=\left[\begin{array}{lll}
\rho_{1} M & Q_{2} N & \rho_{2} T
\end{array}\right], \quad \Gamma=\operatorname{diag}\left\{-Q_{1} Z_{1},-Q_{2}\left(Z_{1}+Z_{2}\right),-Q_{2} Z_{2}\right\}, \\
\mathcal{A}_{1}=\left[\begin{array}{llll}
A & A_{d} & 0 & 0
\end{array}\right], \quad \mathcal{A}_{2}=\left[\begin{array}{llll}
A-E & A_{d} & 0 & 0
\end{array}\right], \\
\Lambda_{1}=\left[\begin{array}{llll}
E & -E & 0 & 0
\end{array}\right], \quad \Lambda_{2}=\left[\begin{array}{llll}
0 & E & 0 & -E
\end{array}\right], \\
\Lambda_{3}=\left[\begin{array}{llll}
0 & E & 0 & -E
\end{array}\right], \quad \tilde{d}=\bar{d}-\underline{d}, \quad U=\bar{d} Z_{1}+\tilde{d} Z_{2}, \\
\rho_{1}=\frac{(1-\alpha)^{-\bar{d}}-1}{\alpha}, \quad Q_{2}=\frac{(1-\alpha)^{-\bar{d}}-(1-\alpha)^{-\underline{d}}}{\alpha},
\end{gathered}
$$

and $R \in \mathbf{R}^{n \times n}$ is any constant matrix satisfying $R E=0$ and $\operatorname{rank}(R)=n-r$, then the unforced system (2.1) is regular, causal, and exponentially stable with $\lambda=1 / \sqrt{1-\alpha}$.

Proof. The proof is divided into three parts: (i) To show the regularity and causality; (ii) to show the exponential stability of the difference subsystem; (iii) to show the exponential stability of the algebraic subsystem.

Part (i): Regularity and causality. Since $E$ is regular and $\operatorname{rank}(E)=r$, there exist two nonsingular matrices $G_{1}$ and $H_{1}$ such that

$$
\begin{gathered}
G_{1} E H_{1}=\left[\begin{array}{cc}
I_{r} & 0 \\
0 & 0
\end{array}\right], \quad G_{1} A H_{1}=\left[\begin{array}{cc}
\tilde{A}_{11} & \tilde{A}_{12} \\
\widetilde{A}_{21} & \widetilde{A}_{22}
\end{array}\right], \\
G_{1}^{-T} X G_{1}^{-1}=\left[\begin{array}{cc}
\tilde{X}_{11} & \widetilde{X}_{12} \\
\widetilde{X}_{12}^{T} & \widetilde{X}_{22}
\end{array}\right], \quad H_{1}^{T} M_{1} G_{1}^{-1}=\left[\begin{array}{cc}
\widetilde{M}_{11} & \widetilde{M}_{12} \\
\widetilde{M}_{21} & \widetilde{M}_{22}
\end{array}\right], \quad R G_{1}^{-1}=\left[\begin{array}{ll}
\widetilde{R}_{1} & \widetilde{R}_{2}
\end{array}\right] .
\end{gathered}
$$

Note that $R E=0$ and $\operatorname{rank}(R)=n-r$, it can be verified that $\widetilde{R}_{1}=0, \operatorname{rank}\left(\widetilde{R}_{2}\right)=n-r$ and $\widetilde{R}_{2} \in \mathbf{R}^{n \times(n-r)}$, that is,

$$
R G_{1}^{-1}=\left[\begin{array}{ll}
0 & \widetilde{R}_{2}
\end{array}\right]
$$

From (3.1), it is easy to obtain $\Phi_{11}<0$. In view of $X>0, Q_{l}>0, l=1,2,3, Z_{l}>0, Z_{2}>0, \bar{d}>$ 0 , and $\tilde{d}>0$, it can be further obtained that

$$
-A^{T} R^{T} S R A-(1-\alpha) E^{T} X E+\operatorname{Sym}\left\{M_{1} E\right\}<0 .
$$


Pre- and postmultiplying (3.5) by $H_{1}^{T}$ and $H_{1}$, respectively, and using (3.3) and (3.4), it is obtained that

$$
\left[\begin{array}{ll}
\star & \star \\
\star & -\widetilde{A}_{22}^{T} \widetilde{R}_{2}^{T} S \widetilde{R}_{2} \tilde{A}_{22}
\end{array}\right]<0,
$$

where $\star$ represents matrices that are not relevant in the following discussion. Thus,

$$
-\widetilde{A}_{22}^{T} \widetilde{R}_{2}^{T} S \widetilde{R}_{2} \widetilde{A}_{22}<0
$$

Now, we assume that the matrix $\tilde{A}_{22}$ is singular, then, there exists a vector $\eta \in \mathbf{R}^{n-r}$ and $\eta \neq 0$ such that $\widetilde{A}_{22} \eta=0$. Pre- and postmultiplying (3.7) by $\eta^{T}$ and $\eta$, respectively, result in $\eta^{T} \widetilde{A}_{22}^{T} \widetilde{R}_{2}^{T} S \widetilde{R}_{2} \widetilde{A}_{22} \eta=0$. Then, it is easy to see that (3.7) is a contradiction since $\eta^{T} \widetilde{A}_{22}^{T} \tilde{R}_{2}^{T} S \widetilde{R}_{2} \widetilde{A}_{22} \eta>0$. Thus, $\tilde{A}_{22}$ is nonsingular, which implies that the unforced system (2.1) is regular and causal by Definition 2 and Theorem 1 in [4].

Part (ii): Exponential stability of the difference subsystem. From [29], the regularity and causality of the unforced system (2.1) imply that there exist two nonsingular matrices $G_{2}$ and $\mathrm{H}_{2}$ such that

$$
\bar{E}=G_{2} E H_{2}=\left[\begin{array}{cc}
I_{r} & 0 \\
0 & 0
\end{array}\right], \quad \bar{A}=G_{2} A H_{2}=\left[\begin{array}{cc}
\bar{A}_{11} & 0 \\
0 & I_{n-r}
\end{array}\right] .
$$

According to (3.8), define

$$
\begin{gathered}
\bar{A}_{d}=G_{2} A_{d} H_{2}=\left[\begin{array}{ll}
\bar{A}_{d 11} & \bar{A}_{d 12} \\
\bar{A}_{d 21} & \bar{A}_{d 22}
\end{array}\right], \quad \bar{X}=G_{2}^{-T} X G_{2}^{-1}=\left[\begin{array}{ll}
\bar{X}_{11} & \bar{X}_{12} \\
\bar{X}_{12}^{T} & \bar{X}_{22}
\end{array}\right], \\
\bar{Q}_{l}=H_{2}^{T} Q_{l} H_{2}=\left[\begin{array}{ll}
\bar{Q}_{l 11} & \bar{Q}_{l 12} \\
\bar{Q}_{l 12}^{T} & \bar{Q}_{l 22}
\end{array}\right], \quad \bar{Z}_{v}=G_{2}^{-T} Z_{v} G_{2}^{-1}=\left[\begin{array}{ll}
\bar{Z}_{v 11} & \bar{Z}_{v 12} \\
\bar{Z}_{v 12}^{T} & \bar{Z}_{v 22}
\end{array}\right], \\
\bar{M}_{v}=H_{2}^{T} M_{v} G_{2}^{-1}=\left[\begin{array}{ll}
\bar{M}_{v 11} & \bar{M}_{v 12} \\
\bar{M}_{v 21} & \bar{M}_{v 22}
\end{array}\right], \quad \bar{N}_{v}=H_{2}^{T} N_{v} G_{2}^{-1}=\left[\begin{array}{ll}
\bar{N}_{v 11} & \bar{N}_{v 12} \\
\bar{N}_{v 21} & \bar{N}_{v 22}
\end{array}\right], \\
\bar{T}_{v}=H_{2}^{T} T_{v} G_{2}^{-1}=\left[\begin{array}{ll}
\bar{T}_{v 11} & \bar{T}_{v 12} \\
\bar{T}_{v 21} & \bar{T}_{v 22}
\end{array}\right], \quad \bar{R}=R G_{2}^{-1}=\left[\begin{array}{ll}
0 & \bar{R}_{2}
\end{array}\right], \quad l=1,2,3, v=1,2 .
\end{gathered}
$$

By using Schur complement on (3.1), we get

$$
\left[\begin{array}{cc}
\Phi_{11}^{\prime} & \Phi_{12}^{\prime} \\
* & \Phi_{22}^{\prime}
\end{array}\right]<0,
$$


where

$$
\begin{gathered}
\Phi_{11}^{\prime}=-\mathrm{A}^{T} R^{T} S R A-(1-\alpha) E^{T} P E+(1+\tilde{d}) Q_{1}+Q_{2}+Q_{3}+\operatorname{Sym}\left\{M_{1} E\right\}, \\
\Phi_{12}^{\prime}=-A^{T} R^{T} S R A_{d}-M_{1} E+N_{1} E-T_{1} E+E^{T} M_{2}^{T}, \\
\Phi_{22}^{\prime}=-A_{d}^{T} R^{T} S R A_{d}-(1-\alpha)^{\bar{d}} Q_{1}+\operatorname{Sym}\left\{-M_{2} E+N_{2} E-T_{2} E\right\} .
\end{gathered}
$$

Substituting (3.8) and (3.9) into the above inequality, pre- and postmultiplying by $\operatorname{diag}\left\{H_{2}^{T}, H_{2}^{T}\right\}$ and $\operatorname{diag}\left\{H_{2}, H_{2}\right\}$, respectively, and using Schur complement yields

$$
\left[\begin{array}{cc}
-\bar{R}_{2}^{T} S \bar{R}_{2}+(1+\tilde{d}) \bar{Q}_{122}+\bar{Q}_{222}+\bar{Q}_{322} & -\bar{R}_{2}^{T} S \bar{R}_{2} \bar{A}_{d 22} \\
* & -(1-\alpha)^{\bar{d}} \bar{Q}_{122}-\bar{A}_{d 22}^{T} \bar{R}_{2}^{T} S \bar{R}_{2} \bar{A}_{d 22}
\end{array}\right]<0
$$

Pre- and postmultiplying this inequality by $\left[-\bar{A}_{d 22}^{T} I\right]$ and its transpose, respectively, and noting $\bar{A}_{d 22}^{T}\left(\bar{Q}_{222}+\bar{Q}_{322}\right) \bar{A}_{d 22} \geq 0$, we have

$$
\bar{A}_{d 22}^{T} \bar{Q}_{122} \bar{A}_{d 22}-\frac{(1-\alpha)^{\bar{d}}}{1+\widetilde{d}} \bar{Q}_{122}<0
$$

Therefore, according to Lemma 2.6, there exist constants $x=\sqrt{\lambda_{\max }\left(\bar{Q}_{122}\right) / \lambda_{\min }\left(\bar{Q}_{122}\right)}$ and $\lambda=-\ln (1-\alpha)^{\bar{d} / 2}+\ln (1+\widetilde{d})^{1 / 2}$ such that

$$
\left\|\bar{A}_{d 22}^{i}\right\| \leq x e^{-\lambda i}, \quad i=1,2, \ldots
$$

Let $\xi(k)=H_{2}^{-1} x(k)=\left[\xi_{1}^{T}(k) \xi_{2}^{T}(k)\right]^{T}$, where $\xi_{1}(k) \in \mathbf{R}^{r}$ and $\xi_{2}(k) \in \mathbf{R}^{n-r}$. Then, the unforced system (2.1) is r.s.e. to the following one:

$$
\begin{gathered}
\xi_{1}(k+1)=\bar{A}_{11} \xi_{1}(k)+\bar{A}_{d 11} \xi_{1}(k-d(k))+\bar{A}_{d 12} \xi_{2}(k-d(k)), \\
0=\xi_{2}(k)+\bar{A}_{d 21} \xi_{1}(k-d(k))+\bar{A}_{d 22} \xi_{2}(k-d(k)) .
\end{gathered}
$$

Now, choose the following Lyapunov-Krasovskii function:

$$
V\left(\xi_{k}\right)=\sum_{s=1}^{4} V_{s}\left(\xi_{k}\right)
$$


where

$$
\begin{aligned}
& V_{1}\left(\xi_{k}\right)=\xi_{1}^{T}(k) \bar{X}_{11} \xi_{1}(k)=\xi^{T}(k) \bar{E}^{T} \overline{X E} \xi(k), \\
& V_{2}\left(\xi_{k}\right)=\sum_{l=k-d(k)}^{k-1} \xi^{T}(l)(1-\alpha)^{k-1-l} \bar{Q}_{1} \xi(l)+\sum_{\theta=-\bar{d}+1}^{-\underline{d}} \sum_{l=k+\theta}^{k-1} \xi^{T}(l)(1-\alpha)^{k-1-l} \bar{Q}_{1} \xi(l), \\
& V_{3}\left(\xi_{k}\right)=\sum_{l=k-\underline{d}}^{k-1} \xi^{T}(l)(1-\alpha)^{k-1-l} \bar{Q}_{2} \xi(l)+\sum_{l=k-\bar{d}}^{k-1} \xi^{T}(l)(1-\alpha)^{k-1-l} \bar{Q}_{3} \xi(l), \\
& V_{4}\left(\xi_{k}\right)=\sum_{\theta=-\bar{d}} \sum_{l=k+\theta}^{k-1} \eta^{T}(l) \bar{E}^{T}(1-\alpha)^{k-1-l} \bar{Z}_{1} \bar{E} \eta(l)+\sum_{\theta=-\bar{d}}^{-\frac{d}{d}-1} \sum_{l=k+\theta}^{k-1} \eta^{T}(l) \bar{E}^{T}(1-\alpha)^{k-1-l} \bar{Z}_{2} \bar{E} \eta(l),
\end{aligned}
$$

with $\xi_{k}(s)=\xi(k+s), \forall s \in \mathbf{I}[-\bar{d}, 0]$ and $\eta(k)=\xi(k+1)-\xi(k)$. Define

$$
\Delta V\left(\xi_{k}\right)=\sum_{s=1}^{4}\left[\Delta V_{s}\left(\xi_{k}\right)\right]=\sum_{s=1}^{4}\left[V_{s}\left(\xi_{k+1}\right)-(1-\alpha) V_{s}\left(\xi_{k}\right)\right]
$$

Then, it follows from (2.1) that

$$
\begin{aligned}
\Delta V_{1}\left(\xi_{k}\right)= & \xi^{T}(k+1) \bar{E}^{T} \bar{X} \bar{E} \xi(k+1)-(1-\alpha) \xi^{T}(k) \bar{E}^{T} \bar{X} \bar{E} \xi(k) \\
= & \left(\bar{A} \xi(k)+\bar{A}_{d} \xi(k-d(k))\right)^{T} \bar{X}\left(\bar{A} \xi(k)+\bar{A}_{d} \xi(k-d(k))\right)-(1-\alpha) \xi^{T}(k) \bar{E}^{T} \bar{X} \bar{E} \xi(k), \\
\Delta V_{2}\left(\xi_{k}\right) \leq & (1+\tilde{d}) \xi^{T}(k) \bar{Q}_{1} \xi(k)-\xi^{T}(k-d(k))(1-\alpha)^{d(k)} \bar{Q}_{1} \xi(k-d(k)), \\
\Delta V_{3}\left(\xi_{k}\right)= & \xi^{T}(k)\left(\bar{Q}_{2}+\bar{Q}_{3}\right) \xi(k)-\xi^{T}(k-\underline{d})(1-\alpha)^{d} \bar{Q}_{2} \xi(k-\underline{d}) \\
& -\xi^{T}(k-\bar{d})(1-\alpha)^{\bar{d}} \bar{Q}_{3} \xi(k-\bar{d}), \\
\Delta V_{4}\left(\xi_{k}\right)= & \eta^{T}(k) \bar{E}^{T}\left(\bar{d} \bar{Z}_{1}+\tilde{d} \bar{Z}_{2}\right) \bar{E} \eta(k)-\sum_{l=k-\bar{d}}^{k-1} \eta^{T}(l) \bar{E}^{T}(1-\alpha)^{k-l} \bar{Z}_{1} \bar{E} \eta(l) \\
& -\sum_{l=k-\bar{d}}^{k-\underline{d}-1} \eta^{T}(l) \bar{E}^{T}(1-\alpha)^{k-l} \bar{Z}_{2} \bar{E}_{\eta}(l) \\
= & \eta^{T}(k) \bar{E}^{T}\left(\bar{d} \bar{Z}_{1}+\tilde{d} \bar{Z}_{2}\right) \bar{E} \eta(k)-\sum_{l=k-d(k)}^{k-1} \eta^{T}(l) \bar{E}^{T}(1-\alpha)^{k-l} \bar{Z}_{1} \bar{E}_{\eta}(l) \\
& -\sum_{l=k-\bar{d}(k)-1} \eta^{T}(l) \bar{E}^{T}(1-\alpha)^{k-l}\left(\bar{Z}_{1}+\bar{Z}_{2}\right) \bar{E}_{\eta}(l)-\sum_{l=k-d(k)}^{k-d-1} \eta^{T}(l) \bar{E}^{T}(1-\alpha)^{k-l} \bar{Z}_{2} \bar{E} \eta(l) .
\end{aligned}
$$


Let

$$
\psi(k)=\left[\begin{array}{llll}
\xi^{T}(k) & \xi^{T}(k-d(k)) & \xi^{T}(k-\underline{d}) & \xi^{T}(k-\bar{d})
\end{array}\right]^{T} .
$$

Using Lemma 2.5 for the last three terms of $\Delta V_{4}\left(\xi_{k}\right)$, respectively, and noting $\underline{d} \leq d(k) \leq \bar{d}$, we have

$$
\begin{aligned}
& -\sum_{l=k-d(k)}^{k-1} \eta^{T}(l) \bar{E}^{T}(1-\alpha)^{k-l} \bar{Z}_{1} \bar{E} \eta(l) \\
& \leq \rho_{1} \psi^{T}(k) \bar{M} \bar{Z}_{1}^{-1} \bar{M}^{T} \psi(k)+2 \psi^{T}(k) \bar{M} \sum_{l=k-d(k)}^{k-1} \bar{E} \eta(l) \\
& -\sum_{l=k-d(k)}^{k-1}\left[\psi^{T}(k) \bar{M}+(1-\alpha)^{k-l} \eta^{T}(l) \bar{E}^{T} \bar{Z}_{1}\right]\left((1-\alpha)^{k-l} \bar{Z}_{1}\right)^{-1} \\
& \times\left[\bar{M}^{T} \psi(k)+(1-\alpha)^{k-l} \bar{Z}_{1} \bar{E} \eta(l)\right] \\
& -\sum_{l=k-\bar{d}}^{k-d(k)-1} \eta^{T}(l) \bar{E}^{T}(1-\alpha)^{k-l}\left(\bar{Z}_{1}+\bar{Z}_{2}\right) \bar{E} \eta(l) \\
& \leq Q_{2} \psi^{T}(k) \bar{N}\left(\bar{Z}_{1}+\bar{Z}_{2}\right)^{-1} \bar{N}^{T} \psi(k)+2 \psi^{T}(k) \bar{N}^{k-d(k)-1} \sum_{l=k-\bar{d}} \bar{E} \eta(l) \\
& -\sum_{l=k-\bar{d}}^{k-d(k)-1}\left[\psi^{T}(k) \bar{N}+(1-\alpha)^{k-l} \eta^{T}(l) \bar{E}^{T}\left(\bar{Z}_{1}+\bar{Z}_{2}\right)\right]\left((1-\alpha)^{k-l}\left(\bar{Z}_{1}+\bar{Z}_{2}\right)\right)^{-1} \\
& \cdot\left[\bar{N}^{T} \psi(k)+(1-\alpha)^{k-l}\left(\bar{Z}_{1}+\bar{Z}_{2}\right) \bar{E} \eta(l)\right] \\
& -\sum_{l=k-d(k)}^{k-\underline{d}-1} \eta^{T}(l) \bar{E}^{T}(1-\alpha)^{k-l} \bar{Z}_{2} \bar{E} \eta(l) \\
& \leq Q_{2} \psi^{T}(k) \bar{T} \bar{Z}_{2}^{-1} \bar{T}^{T} \psi(k)+2 \psi^{T}(k) \bar{T} \sum_{l=k-d(k)}^{k-d-1} \bar{E} \eta(l) \\
& -\sum_{l=k-d(k)}^{k-\underline{d-1}}\left[\psi^{T}(k) \bar{T}+(1-\alpha)^{k-l} \eta^{T}(l) \bar{E}^{T} \bar{Z}_{2}\right]\left((1-\alpha)^{k-l} \bar{Z}_{2}\right)^{-1} \\
& \times\left[\bar{T}^{T} \psi(k)+(1-\alpha)^{k-l} \bar{Z}_{2} \bar{E} \eta(l)\right] .
\end{aligned}
$$


Note that $\eta(l)=\xi(l+1)-\xi(l)$ provides

$$
\begin{aligned}
& \sum_{l=k-d(k)}^{k-1} \bar{E} \eta(l)=\left[\begin{array}{llll}
\bar{E} & -\bar{E} & 0 & 0
\end{array}\right] \psi(k), \\
& \sum_{l=k-\bar{d}}^{k-d(k)-1} \bar{E} \eta(l)=\left[\begin{array}{llll}
0 & \bar{E} & 0 & -\bar{E}
\end{array}\right] \psi(k), \\
& \sum_{l=k-d(k)}^{k-\underline{d}-1} \bar{E} \eta(l)=\left[\begin{array}{llll}
0 & -\bar{E} & \bar{E} & 0
\end{array}\right] \psi(k) .
\end{aligned}
$$

Also, it follows from $R E=0$ that

$$
\begin{aligned}
0 & =-\xi^{T}(k+1) \bar{E}^{T} \bar{R}^{T} S \overline{R E} \xi^{T}(k+1) \\
& =-\left(\bar{A} \xi(k)+\bar{A}_{d} \xi(k-d(k))\right)^{T} \bar{R}^{T} S \bar{R}\left(\bar{A} \xi(k)+\bar{A}_{d} \xi(k-d(k))\right)
\end{aligned}
$$

Then, substituting (3.19)-(3.24) into (3.18), using (3.25), and noting $1-\alpha>0, \bar{Z}_{1}>0$ and $\bar{Z}_{2}>0$, we get

$$
\begin{aligned}
\Delta V\left(\xi_{k}\right) \leq & \psi^{T}(k)\left[\bar{\Phi}+\rho_{1} \bar{M} \bar{Z}_{1}^{-1} \bar{M}^{T}+\rho_{2} \bar{N}\left(Z_{1}+Z_{2}\right)^{-1} \bar{N}^{T}+\rho_{2} \bar{T}^{-1} \bar{Z}_{2}^{T}\right] \psi(k) \\
& -\sum_{l=k-d(k)}^{k-1}\left[\psi^{T}(k) \bar{M}+(1-\alpha)^{k-l} \eta^{T}(l) \bar{E}^{T} \bar{Z}_{1}\right]\left((1-\alpha)^{k-l} \bar{Z}_{1}\right)^{-1} \\
& \times\left[\bar{M}^{T} \psi(k)+(1-\alpha)^{k-l} \overline{Z_{1}} \bar{E} \eta(l)\right] \\
& -\sum_{l=k-\bar{d}}^{k-d(k)-1}\left[\psi^{T}(k) \bar{N}+(1-\alpha)^{k-l} \eta^{T}(l) \bar{E}^{T}\left(\bar{Z}_{1}+\bar{Z}_{2}\right)\right]\left[(1-\alpha)^{k-l}\left(\bar{Z}_{1}+\bar{Z}_{2}\right)\right]^{-1} \\
& \cdot\left[\bar{N}^{T} \psi(k)+(1-\alpha)^{k-l}\left(\bar{Z}_{1}+\bar{Z}_{2}\right) \bar{E} \eta(l)\right] \\
& -\sum_{l=k-d(k)}^{k-d-1}\left[\psi^{T}(k) \bar{T}+(1-\alpha)^{k-l} \eta^{T}(l) \bar{E}^{T} \bar{Z}_{2}\right]\left((1-\alpha)^{k-l} \bar{Z}_{2}\right)^{-1} \\
& \times\left[\bar{T}^{T} \psi(k)+(1-\alpha)^{k-l} \bar{Z}_{2} \bar{E} \eta(l)\right] \\
\leq & \psi^{T}(k)\left[\bar{\Phi}+\rho_{1} \bar{M} \bar{Z}_{1}^{-1} \bar{M}^{T}+\rho_{2} \bar{N}\left(Z_{1}+Z_{2}\right)^{-1} \bar{N}^{T}+\rho_{2} \bar{T} \bar{Z}_{2}^{-1} \bar{T}^{T}\right] \psi(k),
\end{aligned}
$$

where $\bar{\Phi}$ follows the same definition as $\Phi$ defined in (3.1) with $A, A_{d}, X, Q_{l}, Z_{v}, R, M, N$, and $T$ instead of $\bar{A}, \bar{A}_{d}, \bar{X}, \bar{Q}_{l}, \bar{Z}_{v}, \bar{R}, \bar{M}, \bar{N}$, and $\bar{T}$. Performing a congruence transformation on (3.1) by $\operatorname{diag}\left\{H_{2}^{T}, H_{2}^{T}, H_{2}^{T}, H_{2}^{T}, H_{2}^{T}, H_{2}^{T}, H_{2}^{T}\right\}$, and then using the Schur complement implies 
$\bar{\Phi}+\rho_{1} \bar{M} \bar{Z}_{1}^{-1} \bar{M}^{T}+\rho_{2} \bar{N}\left(Z_{1}+Z_{2}\right)^{-1} \bar{N}^{T}+\rho_{2} \bar{T} \bar{Z}_{2}^{-1} \bar{T}^{T}<0$. Thus, it follows from (3.18) and (3.26) that $\Delta V\left(\xi_{k}\right)=V\left(\xi_{k+1}\right)-(1-\alpha) V\left(\xi_{k}\right) \leq 0$, which leads to

$$
V\left(\xi_{k+1}\right) \leq(1-\alpha) V\left(\xi_{k}\right)
$$

By iterative substitutions, inequality (3.27) yields

$$
V\left(\xi_{k}\right) \leq(1-\alpha)^{k} V\left(\xi_{0}\right)
$$

On the other hand, it follows from the Lyapunov functional (3.16) that

$$
\beta_{1}\left\|\xi_{1}(k)\right\|^{2} \leq V\left(\xi_{k}\right), \quad V\left(\xi_{0}\right) \leq \beta_{2}\|\phi\|_{c^{\prime}}^{2}
$$

where

$$
\begin{aligned}
& \beta_{1}=\lambda_{\min }\left(\bar{X}_{11}\right), \\
& \beta_{2}=\lambda_{\max }\left(\bar{X}_{11}\right)+(\bar{d}+\tilde{d}(\bar{d}-1)) \lambda_{\max }\left(\bar{Q}_{1}\right)+\underline{d} \lambda_{\max }\left(\bar{Q}_{2}\right)+\bar{d} \lambda_{\max }\left(\bar{Q}_{3}\right)+\bar{d}^{2} \lambda_{\max }\left(\bar{Z}_{1}+\bar{Z}_{2}\right),
\end{aligned}
$$

Then, combining (3.28) and (3.29) leads to

$$
\begin{aligned}
\left\|\xi_{1}(k)\right\| & \leq \sqrt{\frac{\beta_{2}}{\beta_{1}}}(1-\alpha)^{k / 2}\|\phi\|_{c} \\
& =\sqrt{\frac{\beta_{2}}{\beta_{1}}} e^{-\left(\ln (1-\alpha)^{-1} / 2\right) k}\|\phi\|_{c^{\prime}}
\end{aligned}
$$

Therefore, the difference subsystem of (3.15) is exponentially stable with a decay rate which is not less than $e^{\ln (1-\alpha)^{-1} / 2}$. The remaining task is to show the exponential stability of the algebraic subsystem.

Part (iii): Exponential stability of the algebraic subsystem. Set $f(k)=-\bar{A}_{d 21} \xi_{1}(k-d(k))$; then, it follows from (3.31) that

$$
\|f(k)\| \leq(1-\alpha)^{-\bar{d} / 2}\left\|\bar{A}_{d 21}\right\| \sqrt{\frac{\beta_{2}}{\beta_{1}}} e^{-\left(\ln (1-\alpha)^{-1} / 2\right) k}\|\phi\|_{c}
$$

Using the second equation in (3.15), (3.14) and Lemma 2.7, one gets

$$
\left\|\xi_{2}(k)\right\| \leq \beta_{3} e^{-\left(\ln (1-\alpha)^{-1} / 2\right) k}\|\phi\|_{c^{\prime}}
$$


where

$$
\beta_{3}=\sqrt{\frac{\lambda_{\max }\left(\bar{Q}_{122}\right)}{\lambda_{\min }\left(\bar{Q}_{122}\right)}}\|\phi\|_{c}+\left(\sqrt{\frac{\lambda_{\max }\left(\bar{Q}_{122}\right)}{\lambda_{\min }\left(\bar{Q}_{122}\right)}} \frac{1}{1-e^{-(1 / 2) \ln (1+\tilde{d})}}+1\right) \sqrt{\frac{\beta_{2}}{\beta_{1}}}(1-\alpha)^{-\bar{d} / 2}\left\|\bar{A}_{d 21}\right\| .
$$

Combining (3.31) and (3.33) yields that

$$
\begin{aligned}
\|x(k)\|^{2} & =\xi^{T}(k) H_{2}^{T} H_{2} \xi(k) \\
& \leq\left\|H_{2}\right\|^{2}\left(\left\|\xi_{1}(k)\right\|^{2}+\left\|\xi_{2}(k)\right\|^{2}\right) \\
& \leq\left(\frac{\beta_{2}}{\beta_{1}}+\beta_{3}^{2}\right) e^{-\left(\ln (1-\alpha)^{-1}\right) k}\|\phi\|_{c}^{2} .
\end{aligned}
$$

Thus, we have

$$
\|x(k)\| \leq \sqrt{\frac{\beta_{2}}{\beta_{1}}+\beta_{3}^{2}} e^{-\left(\ln (1-\alpha)^{-1} / 2\right) k}\|\phi\|_{c}=\sqrt{\frac{\beta_{2}}{\beta_{1}}+\beta_{3}^{2}}\left(\frac{1}{\sqrt{1-\alpha}}\right)^{-k}\|\phi\|_{c}
$$

The proof is completed.

Remark 3.2. Theorem 3.1 is obtained by applying a Lyapunov-Krasovskii function method to both the difference and algebraic subsystems of a discrete-time singular system with timevarying delay. Such a method in dealing with the algebraic subsystem of the discrete-time singular delay system has not been reported in the literature.

Remark 3.3. When $E=I$ in system (2.1), then $R=0$, and we get the exponential stability condition for the standard delay systems $x(k+1)=A x(k)+A_{d} x(k-d(k))$ from (3.1).

Based on the result of Theorem 3.1, we now present the existence conditions of a stabilizing state feedback controller for system (2.1) and the corresponding set of initial condition.

Theorem 3.4. Given constants $0<\alpha<1,0<\underline{d}<\bar{d}, \rho>0, \varepsilon_{1}>0, \varepsilon_{2}>0, \varepsilon_{3}>0, \varepsilon_{4}$, and $\varepsilon_{5}$. If there exist symmetric matrices $X>0, Q_{l}>0, \bar{l}=1,2,3, Z_{v}>0, v=1,2$, and $S$, and matrices 
$M=\left[\begin{array}{llll}M_{1}^{T} & M_{2}^{T} & M_{3}^{T} & M_{4}^{T}\end{array}\right]^{T}, N=\left[\begin{array}{llll}N_{1}^{T} & N_{2}^{T} & N_{3}^{T} & N_{4}^{T}\end{array}\right]^{T}, T=\left[\begin{array}{llll}T_{1}^{T} & T_{2}^{T} & T_{3}^{T} & T_{4}^{T}\end{array}\right]^{T}, \bar{F}$ and $H$ such that the following inequalities hold:

$$
\begin{gathered}
{\left[\begin{array}{ccccccc}
\bar{\Phi}_{j} & \Psi & \Upsilon_{j} & \bar{d} \Pi_{j} & \tilde{d} \Pi_{j} & \Xi & \Upsilon_{j} R^{T} \\
* & \Gamma & 0 & 0 & 0 & 0 & 0 \\
* & * & -2 \varepsilon_{1} I+\varepsilon_{1}^{2} X & 0 & 0 & 0 & 0 \\
* & * & * & -\bar{d}\left(2 \varepsilon_{2} I-\varepsilon_{2}^{2} Z_{1}\right) & 0 & 0 & 0 \\
* & * & * & * & -\tilde{d}\left(2 \varepsilon_{3} I-\varepsilon_{3}^{2} Z_{2}\right) & 0 & 0 \\
* & * & * & * & * & -\rho I-S & 0 \\
* & * & * & * & * & * & -\rho^{-1} I
\end{array}\right]<0, \quad j \in \mathbf{I}\left[1,2^{m}\right],} \\
{\left[\begin{array}{cc}
1 & h_{l} \\
* & E^{T} X E
\end{array}\right] \geq 0, \quad l \in \mathbf{I}[1, m],}
\end{gathered}
$$

where $h_{l}$ denotes the lth row of $H$,

$$
\begin{gathered}
\bar{\Phi}_{j}=\operatorname{diag}\left\{-(1-\alpha) E^{T} X E+(1+\tilde{d}) Q_{1}+Q_{2}+Q_{3},-(1-\alpha)^{\bar{d}} Q_{1},-(1-\alpha)^{\underline{d}} Q_{2},-(1-\alpha)^{\bar{d}} Q_{3}\right\} \\
+\operatorname{Sym}\left\{\Xi R \Upsilon_{j}^{T}+M \Lambda_{1}+N \Lambda_{2}+T \Lambda_{3}\right\}, \\
\Upsilon_{j}=\left[\begin{array}{llll}
A_{j} & A_{d} & 0 & 0
\end{array}\right]^{T}, \quad \Pi_{j}=\left[\begin{array}{llll}
A_{j}-E & A_{d} & 0 & 0
\end{array}\right]^{T} \\
\Xi=\left[\begin{array}{llll}
\varepsilon_{4} I & \varepsilon_{5} I & 0 & 0
\end{array}\right]^{T}, \quad A_{j}=A+B\left(D_{j} \bar{F} E+D_{j}^{-} H\right) .
\end{gathered}
$$

$\Lambda_{1}, \Lambda_{2}, \Lambda_{3}, \Psi, \Gamma$ are defined in (3.1), and $R \in \mathbf{R}^{n \times n}$ is any constant matrix satisfying $R E=0$ with $\operatorname{rank}(R)=n-r$, then system (2.3) is regular, causal, and locally exponentially stable with $\lambda=1 / \sqrt{1-\alpha}$ for any compatible initial condition in the ball

$$
B(\delta)=\left\{\phi \in C_{n, \bar{d}}:\|\phi\|_{c}^{2} \leq \delta\right\}
$$

where

$$
\delta=\frac{1}{\lambda_{\max }\left(E^{T} X E\right)+(\bar{d}+\tilde{d}(\bar{d}-1)) \lambda_{\max }\left(Q_{1}\right)+\underline{d} \lambda_{\max }\left(Q_{2}\right)+\bar{d} \lambda_{\max }\left(Q_{3}\right)+\bar{d}^{2} \lambda_{\max }\left(Z_{1}+Z_{2}\right)} .
$$

Proof. If (3.38) holds, then the ellipsoid $\Omega\left(E^{T} X E, 1\right)$ is included in $\mathcal{L}(H)$ [27] (For more details about the ellipsoids and ellipsoid algorithm, we refer the readers to [32-34]). Suppose that 
$x(k) \in \mathcal{L}(H), \forall k>0$ (will be proved later). Hence, by Lemma 2.8, sat $(\bar{F} E x(k))$ can be expressed as

$$
\operatorname{sat}(\bar{F} E x(k))=\sum_{j=1}^{2^{m}} \alpha_{j}(k)\left(D_{j} \bar{F} E+D_{i}^{-} H\right) x(k)
$$

and it follows that

$$
E x(k+1)=\sum_{j=1}^{2^{m}} \alpha_{j}(k) A_{j} x(k)+A_{d} x(k-d(k)) .
$$

Choose a Lyapunov function as in (3.16), and then, by Theorem 3.1, system (3.43) is regular, causal and locally exponentially stable if there exist symmetric matrices $X>0, Q_{l}>0, l=$ $1,2,3, Z_{v}>0, v=1,2$, and $S$, and matrices $M, N$, and $T$ defined in (3.1) such that

$$
\left[\begin{array}{ll}
\widehat{\Phi} & \Psi \\
* & \Gamma
\end{array}\right]<0
$$

where

$$
\begin{gathered}
\widehat{\Phi}=\operatorname{diag}\left\{-(1-\alpha) E^{T} X E+(1+\tilde{d}) Q_{1}+Q_{2}+Q_{3},-(1-\alpha)^{\bar{d}} Q_{1},-(1-\alpha)^{\underline{d}} Q_{2,}-(1-\alpha)^{\bar{d}} Q_{3}\right\} \\
+\widehat{A}_{1 j}^{T} X \widehat{A}_{1 j}-\widehat{A}_{1 j}^{T} R^{T} S R \widehat{A}_{1 j}+\widehat{A}_{2 j}^{T} U \widehat{A}_{2 j}+\operatorname{Sym}\left\{M \Lambda_{1}+N \Lambda_{2}+T \Lambda_{3}\right\} \\
\widehat{A}_{1 j}=\left[\begin{array}{llll}
\sum_{j=1}^{m} \alpha_{j}(k) A_{j} & A_{d} & 0 & 0
\end{array}\right], \quad \widehat{A}_{2 j}=\left[\begin{array}{llll}
\sum_{j=1}^{m} \alpha_{j}(k) A_{j}-E & A_{d} & 0 & 0
\end{array}\right]
\end{gathered}
$$

Now, provided that inequalities (3.37) hold, then by using Schur complement and Lemma 2.9, one has

$$
\left[\begin{array}{cccccc}
\bar{\Phi}_{j}+\Xi(\rho I+S)^{-1} \Xi^{T} & \Psi & \Upsilon_{j} & \bar{d} \Pi_{j} & \tilde{d} \Pi_{j} & \Upsilon_{j} R^{T} \\
* & \Gamma & 0 & 0 & 0 & 0 \\
* & * & -X^{-1} & 0 & 0 & 0 \\
* & * & * & -\bar{d} Z_{1}^{-1} & 0 & 0 \\
* & * & * & * & -\tilde{d} Z_{2}^{-1} & 0 \\
* & * & * & * & * & -\rho^{-1} I
\end{array}\right]<0, \quad j \in \mathbf{I}\left[1,2^{m}\right] .
$$


This, together with $\alpha_{j}(k) \geq 0$ and $\sum_{j=1}^{2^{m}} \alpha_{j}(k)=1$, implies that

$$
\sum_{j=1}^{2^{m}} \alpha_{j}(k)\left[\begin{array}{cccccc}
\bar{\Phi}_{j}+\Xi(\rho I+S)^{-1} \Xi^{T} & \Psi & \Upsilon_{j} & \bar{d} \Pi_{j} & \tilde{d} \Pi_{j} & \Upsilon_{j} R^{T} \\
* & \Gamma & 0 & 0 & 0 & 0 \\
* & * & -X^{-1} & 0 & 0 & 0 \\
* & * & * & -\bar{d} Z_{1}^{-1} & 0 & 0 \\
* & * & * & * & -\tilde{d} Z_{2}^{-1} & 0 \\
* & * & * & * & * & -\rho^{-1} I
\end{array}\right]<0
$$

that is,

$$
\left[\begin{array}{cccccc}
\sum_{j=1}^{2^{m}} \alpha_{j}(k) \bar{\Phi}_{j}+\Xi(\rho I+S)^{-1} \Xi^{T} & \Psi & \sum_{j=1}^{2^{m}} \alpha_{j}(k) \Upsilon_{j} & \sum_{j=1}^{2^{m}} \alpha_{j}(k) \bar{d} \Pi_{j} & \sum_{j=1}^{2^{m}} \alpha_{j}(k) \tilde{d} \Pi_{j} & \sum_{j=1}^{2^{m}} \alpha_{j}(k) \Upsilon_{j} R^{T} \\
* & \Gamma & 0 & 0 & 0 & 0 \\
* & * & -X^{-1} & 0 & 0 & 0 \\
* & * & * & -\bar{d} Z_{1}^{-1} & 0 & 0 \\
* & * & * & * & -\tilde{d} Z_{2}^{-1} & 0 \\
* & * & * & * & * & -\rho^{-1} I
\end{array}\right]<0 .
$$

Applying Schur complement to (3.49) leads to

$$
\left[\begin{array}{ccccc}
\Lambda_{j} & \Psi & \sum_{j=1}^{2^{m}} \alpha_{j}(k) \Upsilon_{j} & \sum_{j=1}^{2^{m}} \alpha_{j}(k) \bar{d} \Pi_{j} & \sum_{j=1}^{2^{m}} \alpha_{j}(k) \tilde{d} \Pi_{j} \\
* & \Gamma & 0 & 0 & 0 \\
* & * & -X^{-1} & 0 & 0 \\
* & * & * & -\bar{d} Z_{1}^{-1} & 0 \\
* & * & * & * & -\tilde{d} Z_{2}^{-1}
\end{array}\right]<0
$$

where

$$
\Lambda_{j}=\sum_{j=1}^{2^{m}} \alpha_{j}(k) \bar{\Phi}_{j}+\Xi(\rho I+S)^{-1} \Xi^{T}+\rho \sum_{j=1}^{2^{m}} \alpha_{j}(k) \Upsilon_{j} R^{T} \sum_{j=1}^{2^{m}} \alpha_{j}(k) R \Upsilon_{j}^{T}
$$

Rewrite

$$
\sum_{j=1}^{2^{m}} \alpha_{j}(k) \bar{\Phi}_{j}=\sum_{j=1}^{2^{m}} \alpha_{j}(k) \operatorname{Sym}\left\{\Upsilon_{j} R^{T} \Xi^{T}\right\}+\widetilde{\Phi},
$$


where

$$
\begin{aligned}
\widetilde{\Phi}= & \operatorname{diag}\left\{-(1-\alpha) E^{T} X E+(1+\widetilde{d}) Q_{1}+Q_{2}+Q_{3},-(1-\alpha)^{\bar{d}} Q_{1},-(1-\alpha)^{d} Q_{2},-(1-\alpha)^{\bar{d}} Q_{3}\right\} \\
& +\operatorname{Sym}\left\{M \Lambda_{1}+N \Lambda_{2}+T \Lambda_{3}\right\} .
\end{aligned}
$$

From (3.37), it follows that $\rho I+S>0$, and then the following inequality holds from Lemma 2.9

$$
\begin{aligned}
-\sum_{j=1}^{2^{m}} \alpha_{j}(k) \Upsilon_{j} R^{T} S \sum_{j=1}^{2^{m}} \alpha_{j}(k) R \Upsilon_{j}^{T} \leq & \sum_{j=1}^{2^{m}} \alpha_{j}(k) \operatorname{Sym}\left\{\Upsilon_{j} R^{T} \Xi^{T}\right\}+\Xi(\rho I+S)^{-1} \Xi^{T} \\
& +\rho \sum_{j=1}^{2^{m}} \alpha_{j}(k) \Upsilon_{j} R^{T} \sum_{j=1}^{2^{m}} \alpha_{j}(k) R \Upsilon_{j}^{T}
\end{aligned}
$$

which, together with (3.50) and (3.52), implies that

$$
\left[\begin{array}{ccccc}
-\sum_{j=1}^{2_{j}} \alpha_{j}(k) \Upsilon_{j} R^{T} S \sum_{j=1}^{2^{m}} \alpha_{j}(k) R \Upsilon_{j}^{T}+\widetilde{\Phi} & \Psi & \sum_{j=1}^{2^{m}} \alpha_{j}(k) \Upsilon_{j} & \sum_{j=1}^{2^{m}} \alpha_{j}(k) \bar{d} \Pi_{j} & \sum_{j=1}^{2^{m}} \alpha_{j}(k) \widetilde{d} \Pi_{j} \\
* & \Gamma & 0 & 0 & 0 \\
* & * & -X^{-1} & 0 & 0 \\
* & * & * & -\bar{d} Z_{1}^{-1} & 0 \\
* & * & * & * & -\tilde{d} Z_{2}^{-1}
\end{array}\right]<0
$$

Applying Schur complement, it results that inequality (3.55) is equivalent to inequality (3.44). Therefore, if inequalities (3.37) hold, then inequality (3.44) holds, that is, system (3.43) is regular, causal, and exponentially stable, provided that $x(k) \in \mathcal{L}(H), \forall k \geq 0$.

On the other hand, by the proof in Theorem 3.1, inequality (3.44) holding implies $V\left(\xi_{k+1}\right)<(1-\alpha) V\left(\xi_{k}\right)<V\left(\xi_{k}\right)$. Assume the initial condition $x(s)=\phi(s)$ is included in the ball $B(\delta), \forall s \in \mathbf{I}[-\bar{d}, 0]$, and inequalities (3.37) and (3.38) hold. Then, from the definition of $V\left(\xi_{k}\right)$, we get $x^{T}(0) E^{T} X E x(0)=\xi^{T}(0) \bar{E}^{T} \overline{X E} \xi(0) \leq V\left(\xi_{0}\right) \leq \delta^{-1}\|\phi\|_{c}^{2} \leq 1$, that is, $x(0) \in \Omega\left(E^{T} X E, 1\right)$. Since inequalities (3.38) are satisfied, it follows that $x(0) \in \mathcal{L}(H)$. Furthermore, because $V\left(\xi_{k+1}\right)<V\left(\xi_{k}\right)$, we can conclude that $x^{T}(k) E^{T} X E x(k)=\xi^{T}(k) \bar{E}^{T} \overline{X E} \xi(k) \leq V\left(\xi_{k}\right) \leq V\left(\xi_{0}\right) \leq$ $\delta^{-1}\|\phi\|_{c}^{2} \leq 1$, which means that $x(k) \in \mathcal{L}(H), \forall k>0$. This completes the proof.

Remark 3.5. In practice, we may be interested to obtain a ball $ß(\delta)$ of initial condition as large as possible. To maximize the ball of initial condition, the following approximate optimization procedure can be used:

$$
\begin{array}{ll}
\min _{X>0, Q_{1}>0, Q_{2}>0, Q_{3}>0, Z_{1}>0, Z_{2}>0, \bar{F}, H} & r \\
\text { s.t. } & (3.44),(3.45) \text { and } E^{T} X E \leq w_{1}, Q_{1} \leq w_{2}, \\
& Q_{2} \leq w_{3}, Q_{3} \leq w_{4}, Z_{1} \leq w_{5}, Z_{2} \leq w_{6},
\end{array}
$$

where $r=w_{1}+(\bar{d}+\tilde{d}(\bar{d}-1)) w_{2}+\underline{d} w_{3}+\bar{d} w_{4}+\bar{d}^{2} w_{5}+\bar{d}^{2} w_{6}$. 
In the special case that $E=I$, the following corollary directly from Remark 3.3 and Theorem 3.4.

Corollary 3.6. Given constants $0<\alpha<1,0<\underline{d}<\bar{d}, \rho>0, \varepsilon_{1}>0, \varepsilon_{2}>0$, and $\varepsilon_{3}>0$. If there exist matrices $X>0, Q_{l}>0, l=1,2,3, Z_{v}>0, v=1,2, M=\left[\begin{array}{llll}M_{1}^{T} & M_{2}^{T} & M_{3}^{T} & M_{4}^{T}\end{array}\right]^{T}$, $N=\left[\begin{array}{llll}N_{1}^{T} & N_{2}^{T} & N_{3}^{T} & N_{4}^{T}\end{array}\right]^{T}, T=\left[\begin{array}{llll}T_{1}^{T} & T_{2}^{T} & T_{3}^{T} & T_{4}^{T}\end{array}\right]^{T}, F$ and $H$ such that the following hold:

$$
\begin{aligned}
& {\left[\begin{array}{ccccc}
\bar{\Phi}_{j} & \Psi & \Upsilon_{j} & \bar{d} \Pi_{j} & \tilde{d} \Pi_{j} \\
* & \Gamma & 0 & 0 & 0 \\
* & * & -2 \varepsilon_{1} I+\varepsilon_{1}^{2} X & 0 & 0 \\
* & * & * & -\bar{d}\left(2 \varepsilon_{2} I-\varepsilon_{2}^{2} Z_{1}\right) & 0 \\
* & * & * & * & -\tilde{d}\left(2 \varepsilon_{3} I-\varepsilon_{3}^{2} Z_{2}\right)
\end{array}\right]<0, \quad j \in \mathbf{I}\left[1,2^{m}\right],} \\
& {\left[\begin{array}{ll}
1 & h_{l} \\
* & X
\end{array}\right] \geq 0, \quad l \in \mathbf{I}[1, m],}
\end{aligned}
$$

where $h_{l}$ denotes the lth row of $H$,

$$
\begin{gathered}
\bar{\Phi}_{j}=\operatorname{diag}\left\{-(1-\alpha) X+(1+\tilde{d}) Q_{1}+Q_{2}+Q_{3},-(1-\alpha)^{\bar{d}} Q_{1},-(1-\alpha)^{\underline{d}} Q_{2},-(1-\alpha)^{\bar{d}} Q_{3}\right\} \\
+\operatorname{Sym}\left\{M \Lambda_{1}+N \Lambda_{2}+T \Lambda_{3}\right\}, \\
\Upsilon_{j}=\left[\begin{array}{llll}
A_{j} & A_{d} & 0 & 0
\end{array}\right]^{T}, \quad \Pi_{j}=\left[\begin{array}{llll}
A_{j}-I & A_{d} & 0 & 0
\end{array}\right]^{T}, \\
\Lambda_{1}=\left[\begin{array}{llll}
I & -I & 0 & 0
\end{array}\right], \quad \Lambda_{2}=\left[\begin{array}{llll}
0 & I & 0 & -I
\end{array}\right], \\
\Lambda_{3}=\left[\begin{array}{llll}
0 & -I & I & 0
\end{array}\right], \quad A_{j}=A+B\left(D_{j} F+D_{j}^{-} H\right),
\end{gathered}
$$

and $\Psi$ and $\Gamma$ are defined in (3.1), then, under the state feedback $u(k)=F x(k)$, system (2.3) with $E=I$ is locally exponentially stable with $\lambda=1 / \sqrt{1-\alpha}$ for any compatible initial condition in the ball

$$
B(\delta)=\left\{\phi \in C_{n, \bar{d}}:\|\phi\|_{c}^{2} \leq \delta\right\}
$$

where

$$
\delta=\frac{1}{\lambda_{\max }(X)+(\bar{d}+\tilde{d}(\bar{d}-1)) \lambda_{\max }\left(Q_{1}\right)+\underline{d} \lambda_{\max }\left(Q_{2}\right)+\bar{d} \lambda_{\max }\left(Q_{3}\right)+\bar{d}^{2} \lambda_{\max }\left(Z_{1}+Z_{2}\right)} .
$$


Table 1: Maximum allowable decay rates $\lambda$ for different $\bar{d}$ with $\underline{d}=1$.

\begin{tabular}{cccccccc}
\hline $\bar{d}$ & 3 & 4 & 5 & 6 & 7 & 10 & 11 \\
\hline$\lambda$ & 1.2642 & 1.1501 & 1.0936 & 1.0613 & 1.0408 & 1.0101 & 1.0049 \\
\hline
\end{tabular}

Also, as Remark 3.5, the corresponding approximate optimization problem of Corollary 3.6 becomes

$$
\begin{array}{ll}
\lim _{X>0, Q_{1}>0, Q_{2}>0, Q_{3}>0, Z_{1}>0, Z_{2}>0, F, H} & r \\
\text { s.t. } & (3.56),(3.58) \text { and } X \leq w_{1}, Q_{1} \leq w_{2}, \\
& Q_{2} \leq w_{3}, Q_{3} \leq w_{4}, Z_{1} \leq w_{5}, Z_{2} \leq w_{6},
\end{array}
$$

where $r=w_{1}+(\bar{d}+\tilde{d}(\bar{d}-1)) w_{2}+\underline{d} w_{3}+\bar{d} w_{4}+\bar{d}^{2} w_{5}+\bar{d}^{2} w_{6}$.

Remark 3.7. Scalars $\rho, \varepsilon_{f}, f=1,2, \ldots, 5$, in Theorem 3.4 are tuning parameters which need to be given first. In fact, (3.37) and (3.38), for fixed $\alpha>0$ and $0<\underline{d}<\bar{d}$, are bilinear matrix inequalities (BMIs) regarding to these tuning parameters. If one can accept more computational burden, the optimal values of these parameters can be obtained by applying some global optimization algorithms [35] to solve the BMIs.

\section{Numerical Examples}

In this section, two examples are given to show the effectiveness of the proposed method.

Example 4.1. Consider the discrete-time singular time-delay system (2.1) without input and with the following parameters:

$$
E=\left[\begin{array}{ll}
1 & 0 \\
0 & 0
\end{array}\right], \quad A=\left[\begin{array}{cc}
0.5 & 0 \\
0.35 & 0.35
\end{array}\right], \quad A_{d}=\left[\begin{array}{cc}
0.13 & 0 \\
0.2 & 0.1
\end{array}\right] .
$$

Let $R=\left[\begin{array}{ll}0 & 0 \\ 0 & 1\end{array}\right]$. For various delay upper bound $\bar{d}$ and by using Theorem 3.1 in this paper, the maximum allowable values of decay rate $\lambda$, that guarantees the regularity, causality and exponential stability of the system for given delay lower bound $\underline{d}$, are listed in Table 1 . It can be seen from Table 1 that the delay upper bound $\bar{d}$ is related to the decay rate $\lambda$, and a smaller $\lambda$ allows for a larger $\bar{d}$. Figure 1 depicts the simulations results of $x_{1}(k)$ and $x_{2}(k)$ as compared to $1.25^{-k}$ when $d(k)=2+\sin (2.5 \pi k)$ and initial function is $\phi_{k}=[-1,1]^{T}, k=-3,-2, \ldots, 0$. It is clearly observed from Figure 1 that the states $x_{1}(k)$ and $x_{2}(k)$ exponentially converge to zero with a decay rate greater than 1.25 .

Example 4.2. Consider the discrete-time singular time-delay system (2.1) with the following parameters:

$$
E=\left[\begin{array}{cc}
2.9 & 1 \\
0 & 0
\end{array}\right], \quad A=\left[\begin{array}{cc}
1.2 & 0.2 \\
1.2 & 2.2
\end{array}\right], \quad A_{d}=\left[\begin{array}{cc}
0.1 & -0.1 \\
0.3 & -0.2
\end{array}\right], \quad B=\left[\begin{array}{l}
-0.1 \\
-0.4
\end{array}\right]
$$


Table 2: $\delta_{\max }$ and $\bar{F}$ for different values of $\alpha$.

\begin{tabular}{lcc}
\hline$\alpha(\lambda)$ & $\delta_{\max }$ & $\bar{F}$ \\
\hline $0.1(1.0541)$ & 2.1025 & {$[1.36900]$} \\
$0.2(1.1180)$ & 2.0932 & {$[1.32060]$} \\
$0.3(1.1952)$ & 2.0318 & {$[1.21240]$} \\
\hline
\end{tabular}

The time-delay is $1 \leq d(k) \leq 3$. Our purpose is to design the stabilizing controllers for different decay rate $\lambda=1 / \sqrt{1-\alpha}$ and estimate the domain of attraction for the above system.

Let $\rho=0.001, \varepsilon_{1}=2.6, \varepsilon_{2}=67, \varepsilon_{3}=71, \varepsilon_{4}=-0.08, \varepsilon_{5}=0.01$, and $R=\left[\begin{array}{ll}0 & 0 \\ 0 & 5\end{array}\right]$. For various $\alpha$, solving (P1) by using the mincx problem in LMI Toolbox [36], the maximal values of $\delta$, denoted $\delta_{\max }$, and the corresponding controller gains $\bar{F}$ are listed in Table 2. Especially, when $\alpha=0.3$, the feasible solutions to (P1) are

$$
\begin{gathered}
X=\left[\begin{array}{ll}
0.0151 & 0.0118 \\
0.0118 & 0.0288
\end{array}\right], \quad Q_{1}=\left[\begin{array}{ll}
0.0120 & 0.0026 \\
0.0026 & 0.0011
\end{array}\right], \quad Q_{2}=\left[\begin{array}{ll}
0.0180 & 0.0026 \\
0.0026 & 0.0016
\end{array}\right], \\
Q_{3}=\left[\begin{array}{ll}
0.0210 & 0.0015 \\
0.0015 & 0.0016
\end{array}\right], \quad Z_{1}=\left[\begin{array}{ll}
0.0091 & 0.0019 \\
0.0019 & 0.0043
\end{array}\right], \quad Z_{2}=\left[\begin{array}{ll}
0.0097 & 0.0023 \\
0.0023 & 0.0013
\end{array}\right], \\
H=\left[\begin{array}{ll}
1.6287 & 0.5616
\end{array}\right], \quad \bar{F}=\left[\begin{array}{ll}
1.2124 & 0
\end{array}\right],
\end{gathered}
$$

Furthermore, applying the obtained controller and giving a possible time-varying delay $d(k)=2+\sin (2.5 \pi k)$, we obtain the state response of the resulting closed-loop system and the corresponding actuator output as shown in Figures 2 and 3 for the given initial function $\phi_{k}=[-1.6,1]^{T}, k=-3,-2, \ldots, 0$.

For clarity, we give some comments to explain the above simulation results. Firstly, it is easily seen from Table 2 that the smaller $\alpha$, that is, the smaller decay rate $\lambda$, then the larger is the domain $B(\delta)$ for which we guarantee the exponential stability of the saturated system. It shows the tradeoff between the size of the ball of the admissible initial condition and the desired decay rate. Secondly, from the curves in Figures 2 and 3, one can see that despite the partly unknown transition probabilities, either the designed state-feedback or the designed output-feedback controllers are feasible and effective ensuring that the resulting closed-loop systems are stable.

It should be pointed out that the results in [27] cannot be applied to this system, because the system considered here contains state delay.

\section{Conclusions}

The problems of exponential estimates and stabilization for a class of discrete-time singular systems with time-varying state delays and saturating actuators have been investigated in this paper. An exponential admissibility condition has been developed for the system. Also, a saturated state feedback controller and a domain of safe initial conditions have been determined by using LMI optimization-based approach. The effectiveness of the results has been illustrated by numerical examples. Note that in the present work, a quadratic LyapunovKrasovskii function was used for the saturated closed-loop system modeled by differential 


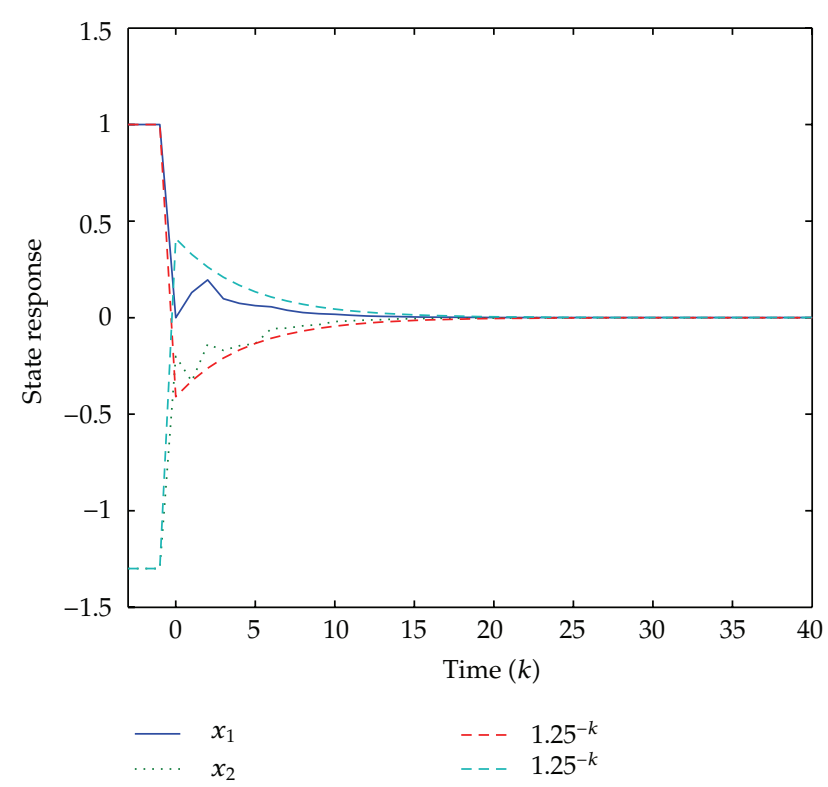

Figure 1: Simulation results of and as compared $1.25^{-k}$.

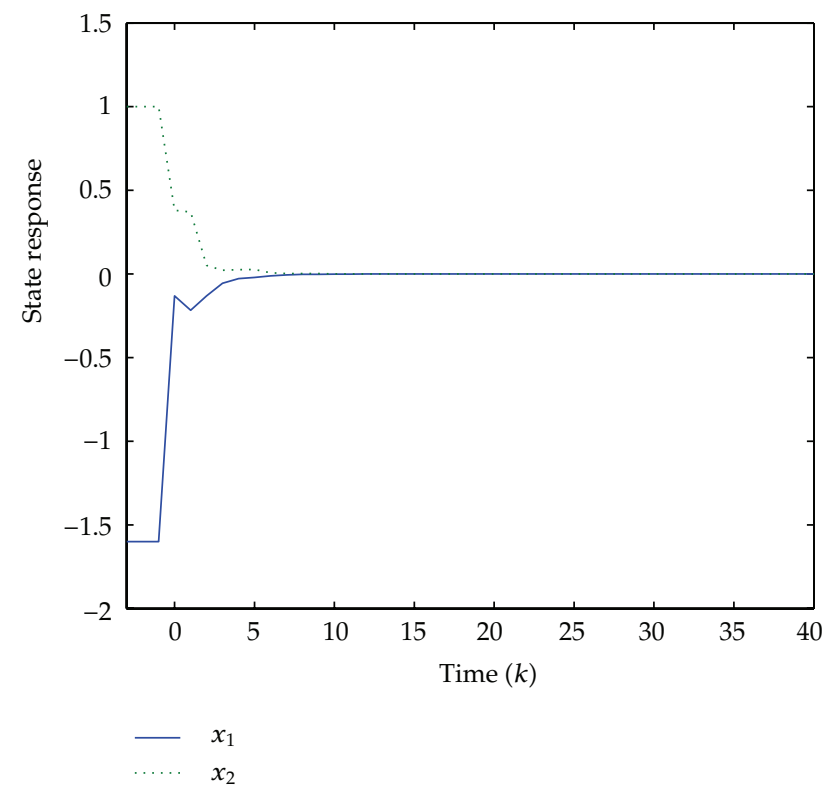

Figure 2: State response of the closed-loop system under saturated control.

inclusion, which may lead to conservatism. Applying the saturation-dependent Lyapunov function approach proposed in [20] to capture the real-time information on the severity of saturation and thus leading to less conservative results will be interesting topics for further research. 


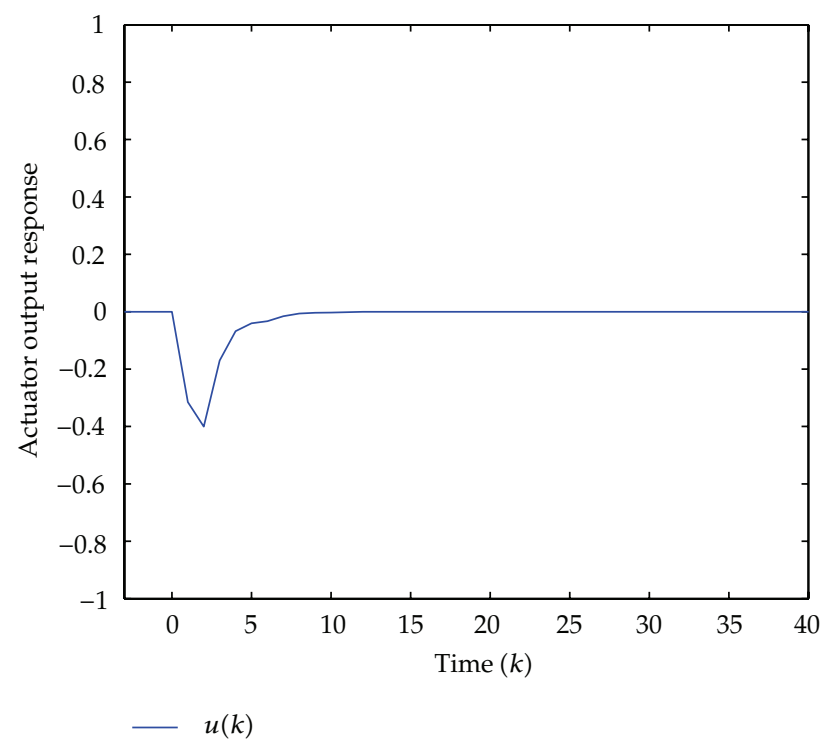

Figure 3: The actuator output under saturated control.

\section{Appendix}

Proof of Lemma 2.5. For given $\zeta(k)$ and $y(k)$ defined in (2.8), the following equation holds

$$
\begin{aligned}
& -\sum_{l=k-d\left(k_{2}\right)}^{k-d\left(k_{1}\right)-1}\left(\zeta^{T}(k) N+\lambda^{k-l} y^{T}(l) E^{T} R\right)\left(\lambda^{k-l} R\right)^{-1}\left(N^{T} \zeta(k)+\lambda^{k-l} R E y(l)\right) \\
& \quad=-\zeta^{T}(k) N R^{-1} N^{T} \xi(k) \sum_{l=k-d\left(k_{2}\right)}^{k-d\left(k_{1}\right)-1} \lambda^{l-k}-2 \zeta^{T}(k) N \sum_{l=k-d\left(k_{2}\right)}^{k-d\left(k_{1}\right)-1} E y(l)-\sum_{l=k-d\left(k_{2}\right)}^{k-d\left(k_{1}\right)-1} y^{T}(l) E^{T} \lambda^{k-l} R E y(l) .
\end{aligned}
$$

Rearranging the above equation yields (2.9). This completes the proof.

Proof of Lemma 2.7. Case $1(k \leq d(k))$. Clearly, $k-d(k) \in[-\bar{d}, 0]$, and then $\|x(k, \phi)\| \leq$ $\|D\|\|\phi\|_{c}+\|f(k)\| \leq x e^{-\lambda}\|\phi\|_{c}+\kappa e^{-\beta k} \leq x e^{-\lambda(k / \bar{d})}\|\phi\|_{c}+\kappa e^{-\beta k} \leq\left(\chi\|\phi\|_{c}+\kappa\right) e^{-r k}$.

Case $2(k>d(k))$. Inspired by the idea in [16], to model the dependency of $x(k)$ on past instances, define

$$
k_{0}=k, \quad k_{i}=k_{i-1}-d\left(k_{i-1}\right), \quad i=1,2, \ldots
$$

Note that $k_{i}<k_{i-1}$ then, by iterative substitutions of (2.10), one gets

$$
x(k, \phi)=(-D)^{k(t)} x\left(k_{k(t)}\right)+\sum_{i=1}^{k(t)-1}(-D)^{i} f\left(k_{i}\right)+f\left(k_{0}\right), \quad k \geq 0,
$$

where $k(t)$ is a positive integer. 
(i) There exists a finite positive integer $k(t)$ such that $0 \leq k_{k(t)-1}$ and $-\bar{d} \leq k_{k(t)}<0$. Then it follows from (A.2) that

$$
k \leq k(t) \bar{d}, \quad k_{i}=k_{0}-\sum_{j=0}^{i-1} d\left(k_{j}\right) \geq k_{0}-i \bar{d}
$$

By (A.3), (A.4), (2.11) and $r=\min \{\lambda / \bar{d}, \beta\}$, the following is true:

$$
\begin{aligned}
\|x(k, \phi)\| & \leq\left\|D^{k(t)}\right\|\|\phi\|_{c}+\sum_{i=1}^{k(t)-1}\left\|D^{i}\right\|\left\|f\left(k_{i}\right)\right\|+\left\|f\left(k_{0}\right)\right\| \\
& \leq x e^{-\lambda k(t)}\|\phi\|_{c}+\sum_{i=1}^{k(t)-1} \chi e^{-\lambda i} \kappa e^{-\beta k_{i}}+\kappa e^{-\beta k_{0}} \\
& \leq x e^{-\lambda(k / \bar{d})}\|\phi\|_{c}+\chi \mathcal{K} \sum_{i=1}^{k(t)-1} e^{-\lambda i} e^{-\beta\left(k_{0}-\sum_{j=0}^{i-1} d\left(k_{j}\right)\right)}+\kappa e^{-\beta k_{0}} \\
& \leq x e^{-\lambda(k / \bar{d})}\|\phi\|_{c}+\chi \mathcal{K} \sum_{i=1}^{k(t)-1} e^{-\lambda i} e^{\beta \bar{i} \bar{d}} e^{-\beta k}+\kappa e^{-\beta k} \\
& \leq\left(x\|\phi\|_{c}+\chi \mathcal{K} \sum_{i=1}^{k(t)-1} e^{(\beta \bar{d}-\lambda) i}+\kappa\right) e^{-r k} \\
& \leq\left(x\|\phi\|_{c}+\chi \mathcal{K} \frac{1}{1-e^{\beta \bar{d}-\lambda}}+\kappa\right) e^{-r k} .
\end{aligned}
$$

(ii) $k_{0}-\sum_{j=0}^{i-1} d\left(k_{j}\right) \geq 0$ for all positive integer $i$ with $i \geq 1$. Since $d(k) \geq 0$, the sequence $\left\{k_{0}-\sum_{j=0}^{i-1} d\left(k_{j}\right)\right\}_{i=1}^{+\infty}$ is monotonically decreased and has a greatest lower bound $\epsilon$ with $\epsilon \geq 0$; that is $\lim _{i \rightarrow+\infty} k_{0}-\sum_{j=0}^{i-1} d\left(k_{j}\right)=\epsilon$. Noting $\lambda>0$, we have $\lim _{i \rightarrow+\infty} e^{-\lambda k_{i}}\left\|x_{k_{i}}\right\|=0$ [8]. Then, similar to the process to derive (A.5), the results in the lemma holds.

\section{Acknowledgment}

This work was supported by the National Natural Science Foundation of China under Grant 60904020, Open Fund of Key Laboratory of Measurement and Control of CSE (no. MCCSE2012A06), Ministry of Education, China, Southeast University, and the Scientific Research Foundation of Nanjing University of Posts and Telecommunications (NY210080).

\section{References}

[1] J. K. Hale and S. M. Verduyn Lunel, Introduction to Functional-Differential Equations, vol. 99 of Applied Mathematical Sciences, Springer, New York, NY, USA, 1993.

[2] S. Xu, P. Van Dooren, R. Ştefan, and J. Lam, "Robust stability and stabilization for singular systems with state delay and parameter uncertainty," IEEE Transactions on Automatic Control, vol. 47, no. 7, pp. 1122-1128, 2002. 
[3] Z. Wu, H. Su, and J. Chu, "Robust stabilization for uncertain discrete singular systems with state delay," International Journal of Robust and Nonlinear Control, vol. 18, no. 16, pp. 1532-1550, 2008.

[4] X. Ji, Z. Yang, and H. Su, "Robust stabilization for uncertain discrete singular time-delay systems," Asian Journal of Control, vol. 12, no. 2, pp. 216-222, 2010.

[5] Z. Du, Q. Zhang, and G. Chang, "Delay-dependent robust $H_{\infty}$ control for uncertain descriptor systems with multiple state delays," Optimal Control Applications \& Methods, vol. 31, no. 4, pp. 375-387, 2010.

[6] J. H. Kim, "Delay-dependent robust $H_{\infty}$ control for discrete-time uncertain singular systems with interval time-varying delays in state and control input," Journal of the Franklin Institute, vol. 347, no. 9, pp. 1704-1722, 2010.

[7] S. Zhu, Z. Li, and C. Zhang, "Delay decomposition approach to delay-dependent stability for singular time-delay systems," IET Control Theory E Applications, vol. 4, no. 11, pp. 2613-2620, 2010.

[8] F. Li and X. Zhang, "A delay-dependent bounded real lemma for singular LPV systems with timevariant delay," International Journal of Robust and Nonlinear Control, vol. 22, no. 5, pp. 559-574, 2012.

[9] S. Xu, J. Lam, and M. Zhong, "New exponential estimates for time-delay systems," IEEE Transactions on Automatic Control, vol. 51, no. 9, pp. 1501-1505, 2006.

[10] D. Yue and Q.-L. Han, "Robust $H_{\infty}$ filter design of uncertain descriptor systems with discrete and distributed delays," IEEE Transactions on Signal Processing, vol. 52, no. 11, pp. 3200-3212, 2004.

[11] D. Yue and Q. L. Han, "Delay-dependent robust $H_{\infty}$ controller design for uncertain descriptor systems with time-varying discrete and distributed delays," IEE Proceedings: Control Theory and Applications, vol. 152, no. 6, pp. 628-638, 2005.

[12] Z. Shu and J. Lam, "Exponential estimates and stabilization of uncertain singular systems with discrete and distributed delays," International Journal of Control, vol. 81, no. 6, pp. 865-882, 2008.

[13] J. X. Lin, X. L. Zhao, and S. M. Fei, "New delay-range-dependent exponential estimates for singular systems with time-varying delay," International Journal of Control, Automation and Systems, vol. 9, no. 2, pp. 218-227, 2011.

[14] Y. Y. Cao and P. M. Frank, "Analysis and synthesis of nonlinear time-delay systems via fuzzy control approach," IEEE Transactions on Fuzzy Systems, vol. 8, no. 2, pp. 200-211, 2000.

[15] J. J. Rubio and W. Yu, "Stability analysis of nonlinear system identification via delayed neural networks," IEEE Transactions on Circuits and Systems II, vol. 54, no. 2, pp. 161-165, 2007.

[16] A. Haidar and E. K. Boukas, "Exponential stability of singular systems with multiple time-varying delays," Automatica, vol. 45, no. 2, pp. 539-545, 2009.

[17] A. Haidar, E. K. Boukas, S. Xu, and J. Lam, "Exponential stability and static output feedback stabilisation of singular time-delay systems with saturating actuators," IET Control Theory $\mathcal{E}$ Applications, vol. 3, no. 9, pp. 1293-1305, 2009.

[18] T. Hu and Z. Lin, Control Systems with Actuator Saturation: Analysis and Design, Birkhäuser, Basel, Switzerland, 2001.

[19] S. Tarbouriech and J. M. Gomes da Silva, Jr., "Synthesis of controllers for continuous-time delay systems with saturating controls via LMI's," IEEE Transactions on Automatic Control, vol. 45, no. 1, pp. 105-111, 2000.

[20] Y.-Y. Cao and Z. Lin, "Stability analysis of discrete-time systems with actuator saturation by a saturation-dependent Lyapunov function," Automatica, vol. 39, no. 7, pp. 1235-1241, 2003.

[21] L. Zhang, E.-K. Boukas, and A. Haidar, "Delay-range-dependent control synthesis for time-delay systems with actuator saturation," Automatica, vol. 44, no. 10, pp. 2691-2695, 2008.

[22] B. Zhou, Z. Lin, and G. Duan, "Robust global stabilization of linear systems with input saturation via gain scheduling," International Journal of Robust and Nonlinear Control, vol. 20, no. 4, pp. 424-447, 2010.

[23] W. Lan and J. Huang, "Semiglobal stabilization and output regulation of singular linear systems with input saturation," IEEE Transactions on Automatic Control, vol. 48, no. 7, pp. 1274-1280, 2003.

[24] B. Zhou, J. Lam, and G.-R. Duan, "An ARE approach to semi-global stabilization of discrete-time descriptor linear systems with input saturation," Systems E Control Letters, vol. 58, no. 8, pp. 609-616, 2009.

[25] Z. Lin and L. Lv, "Set invariance conditions for singular linear systems subject to actuator saturation," IEEE Transactions on Automatic Control, vol. 52, no. 12, pp. 2351-2355, 2007.

[26] L. Lv and Z. Lin, "Analysis and design of singular linear systems under actuator saturation and $L_{2} / L_{\infty}$ disturbances," Systems \& Control Letters, vol. 57, no. 11, pp. 904-912, 2008.

[27] S. Ma and E. K. Boukas, "Stability and $H_{\infty}$ control for discrete-time singular systems subject to actuator saturation," in Proceedings of the American Control Conference (ACC '09), pp. 1244-1249, Hyatt Regency Riverfront, St Louis, Mo, USA, June 2009. 
[28] X. Ji, Y. Sun, and H. Su, "Analysis and design for singular discrete linear systems subject to actuator saturation," Asian Journal of Control, vol. 13, no. 2, pp. 350-355, 2011.

[29] L. Dai, Singular Control Systems, vol. 118 of Lecture Notes in Control and Information Sciences, Springer, Berlin, Germany, 1989.

[30] V. Kharitonov, S. Mondié, and J. Collado, “Exponential estimates for neutral time-delay systems: an LMI approach," IEEE Transactions on Automatic Control, vol. 50, no. 5, pp. 666-670, 2005.

[31] S. Ma, E.-K. Boukas, and Y. Chinniah, "Stability and stabilization of discrete-time singular Markov jump systems with time-varying delay," International Journal of Robust and Nonlinear Control, vol. 20, no. 5, pp. 531-543, 2010.

[32] F. C. Schweppe, Uncertain Dynamic Systems, Prentice Hall, Englewood Cliffs, NJ, USA, 1973.

[33] W. Yu and J. J. Rubio, "Recurrent neural networks training with stable bounding ellipsoid algorithm," IEEE Transactions on Neural Networks, vol. 20, no. 6, pp. 983-991, 2009.

[34] J. J. Rubio, W. Yu, and A. Ferreyra, "Neural network training with optimal bounded ellipsoid algorithm," Neural Computing and Applications, vol. 18, no. 6, pp. 623-631, 2009.

[35] K. C. Goh, M. G. Safonov, and G. P. Papavassilopoulos, "Global optimization approach for the BMI problem," in Proceedings of the 2nd IEEE International Symposium on Requirements Engineering, pp. 20092014, Lake Buena Vista, Fla, USA, March 1995.

[36] S. Boyd, L. F. Ghaout, E. Feron, and V. Balakrishnan, Linear Matrix Inequality in Systems and Control Theory, SIAM, Philadelphia, Pa, USA, 1994. 


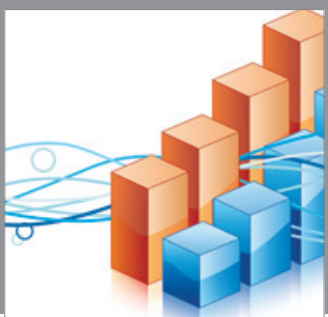

Advances in

Operations Research

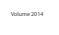

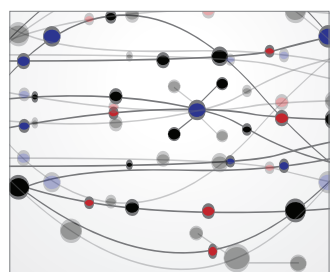

\section{The Scientific} World Journal
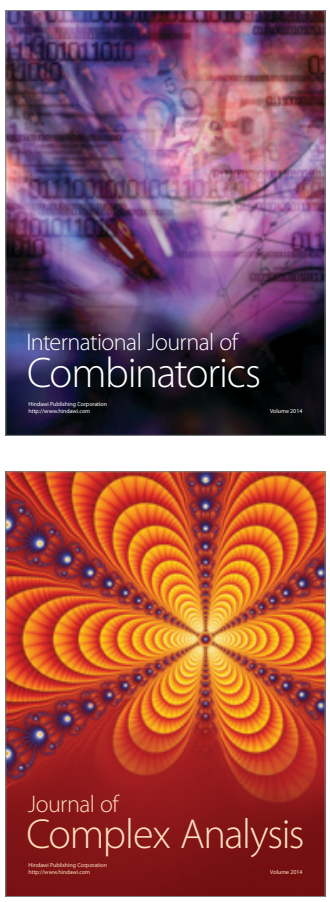

International Journal of

Mathematics and

Mathematical

Sciences
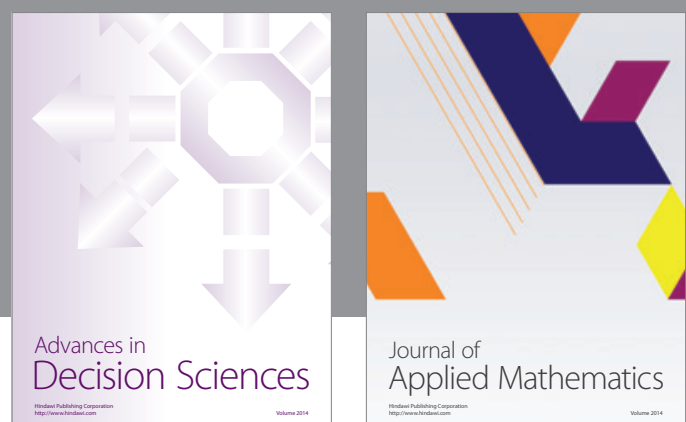

Journal of

Applied Mathematics
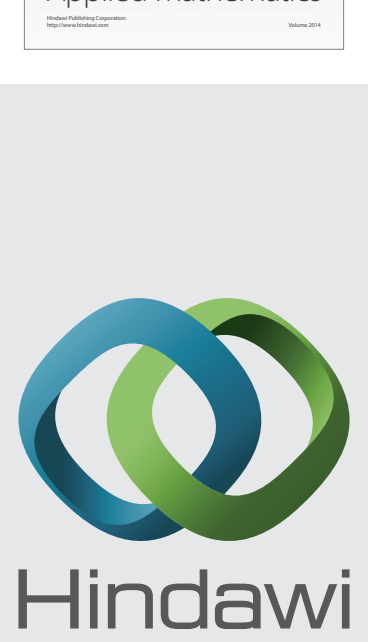

Submit your manuscripts at http://www.hindawi.com
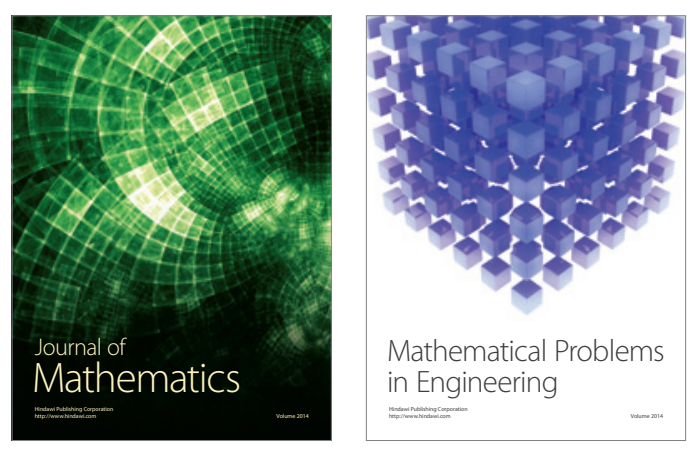

Mathematical Problems in Engineering
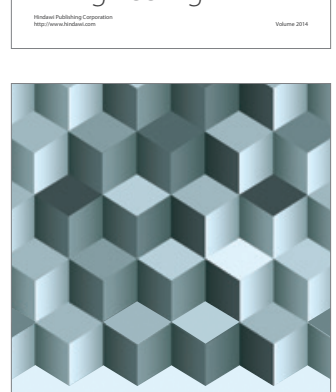

Journal of

Function Spaces
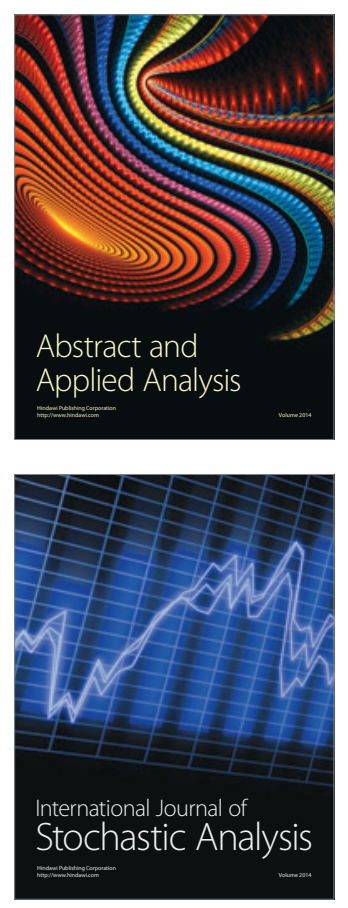

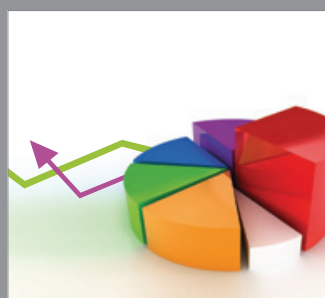

ournal of

Probability and Statistics

Promensencen
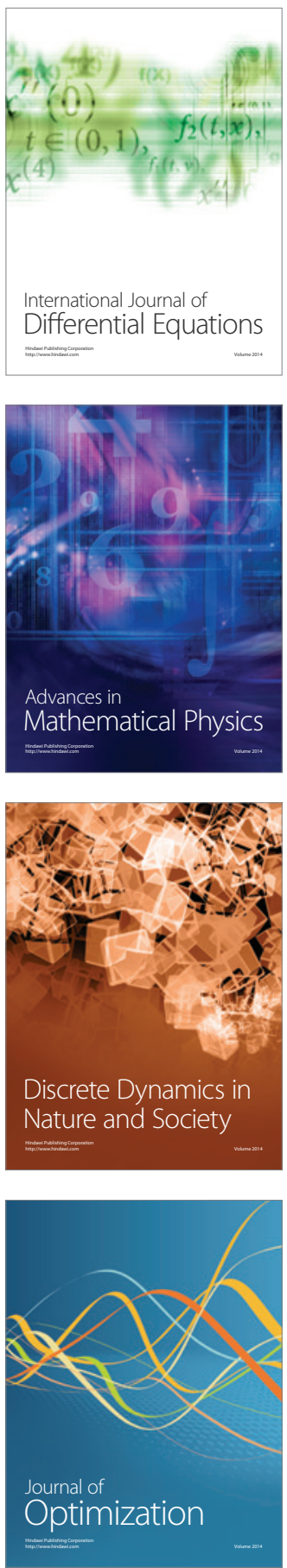